\title{
Rigidity of Log Morphisms
}

\author{
Dedicated to Professor Hironaka on his 7rth birthday
}

By

Atsushi MORIWAKI*

\section{Introduction}

In the paper [6], we proved Kato's conjecture, that is, the finiteness of dominant rational maps in the category of $\log$ schemes as a generalization of Kobayashi-Ochiai theorem [5]. It guarantees the finiteness of $K$-rational points of a certain kind of $\log$ smooth schemes for a big function field $K$, which gives rise to an evidence for Lang's conjecture. In the proof of the above theorem, the most essential part is the rigidity theorem of log morphisms. In this paper, we would like to generalize it to a semistable scheme over an arbitrary noetherian scheme.

Let $f: X \rightarrow S$ be a scheme of finite type over a locally noetherian scheme $S$. We assume that $f: X \rightarrow S$ is a semistable scheme over $S$, namely, $f$ is flat and, for any morphism $\operatorname{Spec}(\Omega) \rightarrow S$ with $\Omega$ an algebraic closed field, the completion of the local ring of $X \times{ }_{S} \operatorname{Spec}(\Omega)$ at every closed point is isomorphic to a ring of the type

$$
\Omega \llbracket X_{1}, \ldots, X_{n} \rrbracket /\left(X_{1} \cdots X_{l}\right)
$$

Let $g: Y \rightarrow S$ be another semistable scheme over $S$, and let $\phi: X \rightarrow Y$ be a morphism over $S$. Let $M_{X}, M_{Y}$ and $M_{S}$ be fine $\log$ structures on $X, Y$ and $S$ respectively. We assume that the morphisms $f: X \rightarrow S$ and $g: Y \rightarrow S$ of schemes extend to $\log$ smooth and integral morphisms $\left(X, M_{X}\right) \rightarrow\left(S, M_{S}\right)$ and $\left(Y, M_{Y}\right) \rightarrow\left(S, M_{S}\right)$ of $\log$ schemes, and that $\phi$ is admissible with respect to $M_{Y} / M_{S}$, i.e., for all $s \in S$ and all irreducible components $V$ of the geometric

\footnotetext{
Communicated by S. Mukai. Received July 24, 2006. Revised February 17, 2007, April $6,2007$.

2000 Mathematics Subject Classification(s): 14A15.

* Department of Mathematics, Faculty of Science, Kyoto University, Kyoto, 606-8502, Japan

e-mail: moriwaki@math.kyoto-u.ac.jp
}

(c) 2008 Research Institute for Mathematical Sciences, Kyoto University. All rights reserved. 
fiber $X \times{ }_{S} \operatorname{Spec}(\overline{\kappa(s)})$ over $s$,

$$
\left.\left(\phi \times_{S} \operatorname{id}_{\operatorname{Spec}(\overline{\kappa(s)})}\right)(V) \nsubseteq \operatorname{Supp}\left(M_{Y} / M_{S}\right)\right|_{Y \times_{S} \operatorname{Spec}(\overline{\kappa(s)})},
$$

where

$$
\operatorname{Supp}\left(M_{Y} / M_{S}\right)=\left\{y \in Y \mid M_{S, \overline{g(y)}} \times \mathcal{O}_{Y, \bar{y}}^{\times} \rightarrow M_{Y, \bar{y}} \text { is not surjective }\right\} .
$$

The following theorem is one of the main results of this paper.

Theorem A (Rigidity theorem). If we have log morphisms

$$
(\phi, h):\left(X, M_{X}\right) \rightarrow\left(Y, M_{Y}\right) \quad \text { and } \quad\left(\phi, h^{\prime}\right):\left(X, M_{X}\right) \rightarrow\left(Y, M_{Y}\right)
$$

over $\left(S, M_{S}\right)$ as extensions of $\phi: X \rightarrow Y$, then $h=h^{\prime}$.

For the proof of the above theorem, our starting point is the local structure theorem (cf. Theorem 3.1), which asserts the local description of integral and smooth log morphisms of semistable schemes. The case where $S$ is the spectrum of an algebraically closed field is essential for the general local structure theorem. This case was proved in the previous paper [6], which was a generalization of a result due to Olsson [9, Proposition 2.10].

Based on the local structure theorem, the proof of the rigidity theorem is carried out as follows: Clearly we may assume that $S=\operatorname{Spec}(A)$ for some noetherian local ring $(A, m)$. First we establish the theorem in the case where $A$ is an algebraically closed field. This was proved actually in the previous paper [6]. Next, by induction on $n$, we see that the assertion holds for the case $S=\operatorname{Spec}\left(A / m^{n}\right)$. Finally, using the Krull intersection theorem, we can conclude its proof.

In Section 1, we give the definition of semistable schemes and show their elementary properties. In Section 2, we recall several facts concerning log schemes. The local structure theorem is proved in Section 3. It is Section 4 that contains the proof of the rigidity theorem. Several applications of the rigidity theorem will be treated in the forthcoming paper [7].

Finally we would like to express hearty thanks to the referee for a lot of comments to improve the paper.

\section{Conventions and terminology}

We will fix several conventions and terminology of this paper.

1. Throughout this paper, a ring means a commutative ring with the unity. 
2. The set of all natural numbers starting from 0 is denoted by $\mathbb{N}$, that is,

$$
\mathbb{N}=\{0,1,2,3,4,5, \ldots\} .
$$

3. In this paper, the logarithmic structures of schemes means the sense of J.-M Fontaine, L. Illusie, and K. Kato. For the details, we refer to [4]. For a log structure $M_{X}$ on a scheme $X$, we denote the quotient $M_{X} / \mathcal{O}_{X}^{\times}$by $\bar{M}_{X}$.

4. Let $X$ be a scheme and $F$ a sheaf in the étale topology. For a point $x \in X$, the stalk of $F$ at $x$ with respect to the Zariski topology (resp. the étale topology) is denoted by $F_{x}$ (resp. $F_{\bar{x}}$ ).

5. Let $\alpha: M_{X} \rightarrow \mathcal{O}_{X}$ be a $\log$ structure on a scheme $X$. For $x \in X$, an element $p \in \bar{M}_{X, \bar{x}}$ is said to be regular if there is $m \in M_{X, \bar{x}}$ such that $p$ coincides with $m$ modulo $\mathcal{O}_{X, \bar{x}}^{\times}$and $\alpha(m)$ is a regular element of $\mathcal{O}_{X, \bar{x}}$, that is, the homomorphism $\mathcal{O}_{X, \bar{x}} \rightarrow \mathcal{O}_{X, \bar{x}}$ given by $\phi \mapsto \alpha(m) \phi$ is injective. Note that the regularity of $p$ does not depend on the choice of $m$.

6. Throughout this paper, a monoid is a commutative monoid with the unity. The binary operation of a monoid is often written additively. We say a monoid $P$ is finitely generated if there are $p_{1}, \ldots, p_{n}$ such that $P=\mathbb{N} p_{1}+\cdots+\mathbb{N} p_{r}$. Moreover $P$ is said to be integral if whenever $x+z=y+z$ for some elements $x, y, z \in P$, we have $x=y$. An integral and finitely generated monoid is said to be fine. We say $P$ is sharp if whenever $x+y=0$ for some $x, y \in P$, then $x=y=0$. For a sharp monoid $P$, an element $x$ of $P$ is said to be irreducible if whenever $x=y+z$ for some $y, z \in P$, then either $y=0$ or $z=0$. A homomorphism $f: Q \rightarrow P$ of monoids is said to be integral if it is injective and an equation

$$
f(q)+p=f\left(q^{\prime}\right)+p^{\prime} \quad\left(p, p^{\prime} \in P, q, q^{\prime} \in Q\right)
$$

implies that $p=f\left(q_{1}\right)+p^{\prime \prime}$ and $p^{\prime}=f\left(q_{2}\right)+p^{\prime \prime}$ for some $p^{\prime \prime} \in P$ and some $q_{1}, q_{2} \in Q$ with $q+q_{1}=q^{\prime}+q_{2}$. Further we say an injective homomorphism $f: Q \rightarrow P$ splits if there is a submonoid $N$ of $P$ such that the homomorphism $f(Q) \times N \rightarrow P$ given by $(x, y) \mapsto x+y$ is an isomorphism.

7. Let $f: Q \rightarrow P$ and $g: Q \rightarrow R$ be homomorphisms of monoids. The integral tensor product $P \bar{\otimes}_{Q} R$ of $P$ and $R$ over $Q$ is defined as follows: Let us consider a relation $\sim$ on $P \times R$ given by

$$
(p, r) \sim\left(p^{\prime}, r^{\prime}\right) \Longleftrightarrow\left(f(q), g\left(q^{\prime}\right)\right)+(p, r)=\left(f\left(q^{\prime}\right), g(q)\right)+\left(p^{\prime}, r^{\prime}\right) \text { for some } q, q^{\prime} \in Q .
$$

It is easy to see that $\sim$ is an equivalence relation on $P \times R$. We set

$$
P \bar{\otimes}_{Q} R=P \times R / \sim .
$$


Note that $P \bar{\otimes}_{Q} R$ is a monoid in the natural way and it is integral if so are $P$ and $R$ (for more details, see [7]).

8. Let $X$ be a set. We denote the set of all maps $X \rightarrow \mathbb{N}$ by $\mathbb{N}^{X}$. For $T \in \mathbb{N}^{X}$, $\operatorname{Supp}(T)$ is given by $\{x \in X \mid T(x)>0\}$. Moreover, for $T, T^{\prime} \in \mathbb{N}^{X}$,

$$
T \leq T^{\prime} \stackrel{\text { def }}{\Longleftrightarrow} T(x) \leq T^{\prime}(x) \forall x \in X .
$$

In the case where $X=\{1, \ldots, n\}, \mathbb{N}^{X}$ is sometimes denoted by $\mathbb{N}^{n}$.

9. Let $M$ be a monoid, $X$ a finite subset of $M$ and $T \in \mathbb{N}^{X}$. For simplicity, $\sum_{x \in X} T(x) x$ is often denoted by $T \cdot X$. If we use the product symbol for the binary operation of the monoid $M$, then $\prod_{x \in X} x^{T(x)}$ is written by $X^{T}$. In particular, if $X=\left\{X_{1}, \ldots, X_{n}\right\}$ and $I \in \mathbb{N}^{n}$, then $I \cdot X$ and $X^{I}$ means $\sum_{i=1}^{n} I(i) X_{i}$ and $\prod_{i=1}^{n} X_{i}^{I(i)}$ respectively according to a way of the binary operator of $M$. For example, let $A$ be a ring and let $R$ be either the ring of polynomials of $n$-variables over $A$, or the ring of formal power series of $n$-variables over $A$, that is, $R=A\left[X_{1}, \ldots, X_{n}\right]$ or $A \llbracket X_{1}, \ldots, X_{n} \rrbracket$. Note that $R$ is a monoid with respect to the ring multiplication. As explained in the above, for $I \in \mathbb{N}^{n}$, the monomial $X_{1}^{I(1)} \cdots X_{n}^{I(n)}$ is denoted by $X^{I}$.

10. Let $f: Q \rightarrow P$ be an integral homomorphism of fine and sharp monoids. In the following, the binary operators of monoids are written in the additive way. For a finite subset $\sigma$ of $P, q_{0} \in Q$ and $\Delta, B \in \mathbb{N}^{\sigma}$, we say $P$ has a semistable structure $\left(\sigma, q_{0}, \Delta, B\right)$ over $Q$ (or $P$ is of semistable type $\left(\sigma, q_{0}, \Delta, B\right)$ over $Q$ ) if the following conditions are satisfied:

(1) $q_{0} \neq 0, \operatorname{Supp}(\Delta) \neq \emptyset$ and $\Delta(x)$ is either 0 or 1 for all $x \in \sigma$.

(2) $P$ is generated by $\sigma$ and $f(Q)$ and the natural homomorphism $\mathbb{N}^{\sigma} \rightarrow P$ given by $T \mapsto T \cdot \sigma$ is injective.

(3) $\operatorname{Supp}(\Delta) \cap \operatorname{Supp}(B)=\emptyset$ and $\Delta \cdot \sigma=f\left(q_{0}\right)+B \cdot \sigma$.

(4) If we have a relation

$$
T \cdot \sigma=f(q)+T^{\prime} \cdot \sigma \quad\left(T, T^{\prime} \in \mathbb{N}^{\sigma}\right)
$$

with $q \neq 0$, then $T(x)>0$ for all $x \in \operatorname{Supp}(\Delta)$.

Let $\mathbb{N} \rightarrow Q \times \mathbb{N}^{\sigma \backslash \operatorname{Supp}(\Delta)}$ and $\mathbb{N} \rightarrow \mathbb{N}^{\operatorname{Supp}(\Delta)}$ be homomorphisms given by $1 \mapsto\left(f\left(q_{0}\right),\left.B\right|_{\sigma \backslash \operatorname{Supp}(\Delta)}\right)$ and $\left.1 \mapsto \Delta\right|_{\operatorname{Supp}(\Delta)}$ respectively. It is known that the natural homomorphism

$$
\left(Q \times \mathbb{N}^{\sigma \backslash \operatorname{Supp}(\Delta)}\right) \bar{\otimes}_{\mathbb{N}} \mathbb{N}^{\operatorname{Supp}(\Delta)} \rightarrow P
$$


is bijective, where $\bar{\otimes}_{\mathbb{N}}$ is the integral tensor product (cf. [6, Proposition 2.2]).

11. Let $(A, m)$ be a local ring. The henselization of $A$ and the completion of $A$ with respect to $m$ are denoted by $A^{h}$ and $\widehat{A}$ respectively.

\section{$\S 1$. Semistable Schemes over a Scheme}

\section{§1.1. Algebraic preliminaries}

In this subsection, we consider several lemmas which will be used later. Let us begin with the following lemma.

Lemma 1.1.1. Let $f:\left(A, m_{A}\right) \rightarrow\left(B, m_{B}\right)$ be a local homomorphism of noetherian local rings such that $f$ induces an isomorphism $A / m_{A} \stackrel{\sim}{\longrightarrow} B / m_{B}$.

(1) Let $x_{1}, \ldots, x_{n}$ be generator of $m_{B}$, i.e., $m_{B}=B x_{1}+\cdots+B x_{n}$. If $\left(B, m_{B}\right)$ is complete, then, for any $b \in B$, there is a sequence

$$
\left\{\alpha_{\left(a_{1}, \ldots, a_{n}\right)}\right\}_{\left(a_{1}, \ldots, a_{n}\right) \in \mathbb{N}^{n}}
$$

of elements of $A$ indexed by $\mathbb{N}^{n}$ with

$$
b=\sum_{\left(a_{1}, \ldots, a_{n}\right) \in \mathbb{N}^{n}} f\left(\alpha_{\left(a_{1}, \ldots, a_{n}\right)}\right) x_{1}^{a_{1}} \cdots x_{n}^{a_{n}} .
$$

(2) Let $x_{1}, \ldots, x_{n}$ be elements of $m_{B}$ with $m_{B}=B x_{1}+\cdots+B x_{n}+m_{A} B$. If $\left(A, m_{A}\right)$ and $\left(B, m_{B}\right)$ are complete, then, for any $b \in B$, there is a sequence

$$
\left\{\alpha_{\left(a_{1}, \ldots, a_{n}\right)}\right\}_{\left(a_{1}, \ldots, a_{n}\right) \in \mathbb{N}^{n}}
$$

of elements of $A$ indexed by $\mathbb{N}^{n}$ with

$$
b=\sum_{\left(a_{1}, \ldots, a_{n}\right) \in \mathbb{N}^{n}} f\left(\alpha_{\left(a_{1}, \ldots, a_{n}\right)}\right) x_{1}^{a_{1}} \cdots x_{n}^{a_{n}} .
$$

Proof. (1) First we claim the following:

Claim 1.1.1.1.

$$
m_{B}^{d} \subseteq \sum_{\substack{\left(a_{1}, \ldots, a_{n}\right) \in \mathbb{N}^{n} \\ a_{1}+\cdots+a_{n}=d}} f(A) x_{1}^{a_{1}} \cdots x_{n}^{a_{n}}+m_{B}^{d+1}
$$

for all $d \geq 0$. 
We prove this claim by induction on $d$. Since $A / m_{A} \simeq B / m_{B}$, we have $B=f(A)+m_{B}$, which means that the assertion holds for $d=0$. Thus

$$
\begin{aligned}
m_{B} & =\left(f(A)+m_{B}\right) x_{1}+\cdots+\left(f(A)+m_{B}\right) x_{n} \\
& \subseteq f(A) x_{1}+\cdots+f(A) x_{n}+m_{B}^{2},
\end{aligned}
$$

which show that the assertion holds for $d=1$, so that we assume $d \geq 2$. By the hypothesis of induction,

$$
\begin{aligned}
m_{B}^{d} & =m_{B} \cdot m_{B}^{d-1} \\
& \subseteq\left(f(A) x_{1}+\cdots+f(A) x_{n}+m_{B}^{2}\right) \cdot\left(\sum_{\substack{\left(a_{1}^{\prime}, \ldots, a_{n}^{\prime}\right) \in \mathbb{N}^{n} \\
a_{1}^{\prime}+\cdots+a_{n}^{\prime}=d-1}} f(A) x_{1}^{a_{1}^{\prime}} \cdots x_{n}^{a_{n}^{\prime}}+m_{B}^{d}\right) \\
& \subseteq \sum_{\substack{\left(a_{1}, \ldots, a_{n}\right) \in \mathbb{N}^{n} \\
a_{1}+\cdots+a_{n}=d}} f(A) x_{1}^{a_{1}} \cdots x_{n}^{a_{n}}+m_{B}^{d+1} .
\end{aligned}
$$

Hence we get the claim.

In order to complete the proof of (1), it is sufficient to see the following claim:

Claim 1.1.1.2. For all $b \in B$, there is a sequence $\left\{b_{d}\right\}_{d=0}^{\infty}$ of $B$ such that

$$
b_{d} \in \sum_{\substack{\left(a_{1}, \ldots, a_{n}\right) \in \mathbb{N}^{n} \\ a_{1}+\cdots+a_{n}=d}} f(A) x_{1}^{a_{1}} \cdots x_{n}^{a_{n}}
$$

and

$$
b-\left(b_{0}+\cdots+b_{d}\right) \in m_{B}^{d+1}
$$

for all $d \geq 0$.

Since $B=f(A)+m_{B}$, we can set $b=b_{0}+c$ with $b_{0} \in f(A)$ and $c \in m_{B}$. We assume that $b_{0}, \ldots, b_{d-1}$ are given. Then, by Claim 1.1.1.1,

$$
b-\left(b_{0}+\cdots+b_{d-1}\right)=b_{d}+c^{\prime},
$$

where $b_{d} \in \sum_{\substack{\left(a_{1}, \ldots, a_{n}\right) \in \mathbb{N}^{n} \\ a_{1}+\cdots+a_{n}=d}} f(A) x_{1}^{a_{1}} \cdots x_{n}^{a_{n}}$ and $c^{\prime} \in m_{B}^{d+1}$. This yields the second claim.

(2) Let us choose $y_{1}, \ldots, y_{r} \in A$ with $m_{A}=y_{1} A+\cdots+y_{r} A$. Then

$$
m_{B}=x_{1} B+\cdots+x_{n} B+f\left(y_{1}\right) B+\cdots+f\left(y_{r}\right) B .
$$


Note that

$$
x_{1}^{a_{1}} \cdots x_{n}^{a_{n}} f\left(y_{1}\right)^{b_{1}} \cdots f\left(y_{r}\right)^{b_{r}}=f\left(y_{1}^{b_{1}} \cdots y_{r}^{b_{r}}\right) x_{1}^{a_{1}} \cdots x_{n}^{a_{n}} .
$$

Therefore, since $\left(A, m_{A}\right)$ is complete, using (1), for any $b \in B$, there is a sequence $\left\{\alpha_{\left(a_{1}, \ldots, a_{n}, b_{1}, \ldots, b_{r}\right)}\right\}_{\left(a_{1}, \ldots, a_{n}, b_{1}, \ldots, b_{r}\right) \in \mathbb{N}^{n} \times \mathbb{N}^{r}}$ with

$$
\begin{array}{r}
b=\sum_{\left(a_{1}, \ldots, a_{n}, b_{1}, \ldots, b_{r}\right) \in \mathbb{N}^{n} \times \mathbb{N}^{r}} f\left(\alpha_{\left(a_{1}, \ldots, a_{n}, b_{1}, \ldots, b_{r}\right)}\right) x_{1}^{a_{1}} \cdots x_{n}^{a_{n}} f\left(y_{1}\right)^{b_{1}} \cdots f\left(y_{r}\right)^{b_{r}} \\
=\sum_{\left(a_{1}, \ldots, a_{n}\right) \in \mathbb{N}^{n}} f\left(\sum_{\left(b_{1}, \ldots, b_{r}\right) \in \mathbb{N}^{r}} \alpha_{\left(a_{1}, \ldots, a_{n}, b_{1}, \ldots, b_{r}\right)} y_{1}^{b_{1}} \cdots y_{r}^{b_{r}}\right) x_{1}^{a_{1}} \cdots x_{n}^{a_{n}} .
\end{array}
$$

Thus we get (2).

Next let us consider the following lemma.

Lemma 1.1.2. Let $(A, m)$ be a noetherian local ring and $T \in \mathbb{N}^{n} \backslash$ $\{(0, \ldots, 0)\}$. Let $G \in m \llbracket X_{1}, \ldots, X_{n} \rrbracket, R=A \llbracket X_{1}, \ldots, X_{n} \rrbracket /\left(X^{T}-G\right)$ and $\pi$ : $A \llbracket X_{1}, \ldots, X_{n} \rrbracket \rightarrow R$ the canonical homomorphism. Then we have the following:

(1) Let $M$ be an $A$-submodule of $A \llbracket X_{1}, \ldots, X_{n} \rrbracket$ given by

$$
M=\left\{\sum_{T \not Z I} a_{I} X^{I} \mid a_{I} \in A\right\}
$$

(cf. Conventions and terminology 8 and 9$)$. If $(A, m)$ is complete, then $\left.\pi\right|_{M}: M \rightarrow R$ is bijective.

(2) $A \llbracket X_{1}, \ldots, X_{n} \rrbracket /\left(X^{T}-G\right)$ is flat over $A$.

Proof. (1) We denote $\pi\left(X_{j}\right)$ by $x_{j}$. First we claim the following:

Claim 1.1.2.1. For $f \in R$, there is a sequence $\left\{F_{i}\right\}_{i=0}^{\infty}$ in $M$ such that $F_{i+1}-F_{i} \in m^{i} \llbracket X_{1}, \ldots, X_{n} \rrbracket$ and $f-\pi\left(F_{i}\right) \in m^{i} R$ for all $i \geq 0$.

We will construct a sequence $\left\{F_{i}\right\}_{i=0}^{\infty}$ inductively. Clearly we may set $F_{0}=0$. We assume that $F_{0}, F_{1}, \ldots, F_{i}$ have been constructed. Then we can set $f-\pi\left(F_{i}\right)=\pi(H)+x^{T} \pi\left(H^{\prime}\right)$ for some $H, H^{\prime} \in m^{i} \llbracket X_{1}, \ldots, X_{n} \rrbracket$ with $H \in M$. Here $x^{T} \pi\left(H^{\prime}\right)=\pi(G) \pi\left(H^{\prime}\right) \in m^{i+1} R$. Thus, if we set $F_{i+1}=F_{i}+H$, then we get our desired $F_{i+1}$. 
The above claim shows that $\left.\pi\right|_{M}$ is surjective. Next let us consider the injectivity of $\left.\pi\right|_{M}$. We assume

$$
\pi\left(\sum_{T \not \subset I} a_{I} X^{I}\right)=0 .
$$

Then there is $H \in A \llbracket X_{1}, \ldots, X_{n} \rrbracket$ with

$$
\sum_{T \not \subset I} a_{I} X^{I}=\left(X^{T}-G\right) H .
$$

Here we set

$$
G=\sum_{I \in \mathbb{N}^{n}} g_{I} X^{I} \quad \text { and } \quad H=\sum_{I \in \mathbb{N}^{n}} h_{I} X^{I}
$$

Then $g_{I} \in m$ for all $I$ and

$$
\sum_{T \nless 1} a_{I} X^{I}=\sum_{I \in \mathbb{N}^{n}} h_{I} X^{T+I}-\sum_{I \in \mathbb{N}^{n}}\left(\sum_{J+J^{\prime}=I} g_{J} h_{J^{\prime}}\right) X^{I} .
$$

On the left hand side of the above equation, there is no term of a form $X^{I+T}$. Thus

$$
h_{I}=\sum_{J+J^{\prime}=I+T} g_{J} h_{J^{\prime}}
$$

for all $I$. Here we claim that $h_{I} \in m^{i}$ for all $i$ and all $I$. We see this fact by induction $i$. First of all, since $g_{I} \in m$ for all $I$, we have $h_{I} \in m$ for all $I$. We assume that $h_{I} \in m^{i}$ for all $I$. By the above equation, we can see that $h_{I} \in m^{i+1}$. By this claim, $h_{I}$ must be zero for all $I$ because $\bigcap_{i \geq 0} m^{i}=0$. Therefore $a_{I}=0$ for all $I$.

(2) Note that the direct product of a family of flat modules over a noetherian ring is again flat (cf. Chase [2]). Therefore, if $(A, m)$ is complete, then the assertion follows from (1). In general, let $\widehat{A}$ be the completion of $A$ and

$$
R^{\prime}=\widehat{A} \llbracket X_{1}, \ldots, X_{n} \rrbracket /\left(X^{T}-G\right) .
$$

Then we have the following commutative diagram:

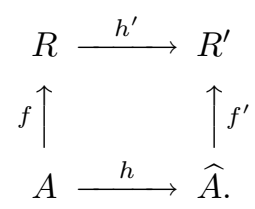

Note that $f^{\prime}, h$ and $h^{\prime}$ are faithfully flat. Thus so is $f$.

We consider an approximation by an étale neighborhood. 
Proposition 1.1.3. Let $\left(A, m_{A}\right)$ be a noetherian local ring essentially of finite type over an excellent discrete valuation ring or a field. Let $f: X \rightarrow$ $\operatorname{Spec}(A)$ be a scheme of finite type over $A$. Let $x$ be a point of $X$ such that $f(x)=m_{A}$ and $A / m_{A}$ is naturally isomorphic to $\mathcal{O}_{X, x} / m_{X, x}$. We assume that there are $F_{1}, \ldots, F_{r} \in A\left[X_{1}, \ldots, X_{n}\right]$ (the polynomial ring of $n$-variables over A) and an isomorphism

$$
\phi: \widehat{A} \llbracket X_{1}, \ldots, X_{n} \rrbracket /\left(F_{1}, \ldots, F_{r}\right) \stackrel{\sim}{\longrightarrow} \widehat{\mathcal{O}}_{X, x}
$$

over $\widehat{A}$ with $\phi\left(\bar{X}_{i}\right) \in \widehat{m}_{X, x}$ for all $i$, where $\bar{X}_{i}=X_{i} \bmod \left(F_{1}, \ldots, F_{r}\right)$. Then there is an étale neighborhood $\left(U, x^{\prime}\right)$ of $X$ at $x$ together with an étale morphism

$$
\rho: U \rightarrow \operatorname{Spec}\left(A\left[T_{1}, \ldots, T_{n}\right] /\left(F_{1}(T), \ldots, F_{r}(T)\right)\right)
$$

such that $\rho\left(x^{\prime}\right)=\left(m_{A}, \bar{T}_{1}, \ldots, \bar{T}_{n}\right)$, where $\bar{T}_{i}=T_{i} \bmod \left(F_{1}(T), \ldots, F_{r}(T)\right)$.

Proof. First note that

$$
F_{1}\left(\phi\left(\bar{X}_{1}\right), \ldots, \phi\left(\bar{X}_{n}\right)\right)=\cdots=F_{r}\left(\phi\left(\bar{X}_{1}\right), \ldots, \phi\left(\bar{X}_{n}\right)\right)=0 .
$$

Thus, by Artin's approximation theorem [1], there are $t_{1}, \ldots, t_{n} \in \mathcal{O}_{X, x}^{h}$ such that

$$
F_{1}\left(t_{1}, \ldots, t_{n}\right)=\cdots=F_{r}\left(t_{1}, \ldots, t_{n}\right)=0
$$

and $t_{i}-\phi\left(\bar{X}_{i}\right) \in \widehat{m}_{X, x}^{2}$ for all $i$. Here we claim the following:

\section{Claim 1.1.3.1.}

$$
\begin{aligned}
\widehat{m}_{X, x} & =\phi\left(\bar{X}_{1}\right) \widehat{\mathcal{O}}_{X, x}+\cdots+\phi\left(\bar{X}_{n}\right) \widehat{\mathcal{O}}_{X, x}+m_{A} \widehat{\mathcal{O}}_{X, x} \\
& =t_{1} \widehat{\mathcal{O}}_{X, x}+\cdots+t_{n} \widehat{\mathcal{O}}_{X, x}+m_{A} \widehat{\mathcal{O}}_{X, x} .
\end{aligned}
$$

Clearly

$$
\widehat{m}_{X, x} \supseteq \phi\left(\bar{X}_{1}\right) \widehat{\mathcal{O}}_{X, x}+\cdots+\phi\left(\bar{X}_{n}\right) \widehat{\mathcal{O}}_{X, x}+m_{A} \widehat{\mathcal{O}}_{X, x} .
$$

Conversely let us pick up $f \in \widehat{m}_{X, x}$. Then we can write $f=\phi\left(\sum_{I} a_{I} \bar{X}^{I}\right)$. If $a_{(0, \ldots, 0)} \in \widehat{A}^{\times}$, then $f$ must be a unit because $f \in a_{(0, \ldots, 0)}+\widehat{m}_{X, x}$. This is a contradiction. Thus $a_{(0, \ldots, 0)} \in m_{A}$, which means that

$$
f \in \phi\left(\bar{X}_{1}\right) \widehat{\mathcal{O}}_{X, x}+\cdots+\phi\left(\bar{X}_{n}\right) \widehat{\mathcal{O}}_{X, x}+m_{A} \widehat{\mathcal{O}}_{X, x}
$$

Therefore we obtain

$$
\widehat{m}_{X, x}=\phi\left(\bar{X}_{1}\right) \widehat{\mathcal{O}}_{X, x}+\cdots+\phi\left(\bar{X}_{n}\right) \widehat{\mathcal{O}}_{X, x}+m_{A} \widehat{\mathcal{O}}_{X, x}
$$


Moreover, since $t_{i}-\phi\left(\bar{X}_{i}\right) \in \widehat{m}_{X, x}^{2}$, we can see that

$$
\widehat{m}_{X, x}=t_{1} \widehat{\mathcal{O}}_{X, x}+\cdots+t_{n} \widehat{\mathcal{O}}_{X, x}+m_{A} \widehat{\mathcal{O}}_{X, x}+\widehat{m}_{X, x}^{2} .
$$

Hence, by Nakayama's lemma, we have our desired result.

Let us choose an étale neighborhood $\left(U, x^{\prime}\right)$ of $X$ at $x$ with the same residue field such that $t_{1}, \ldots, t_{n}$ are defined over $U$. Here let us define a homomorphism

$$
\psi: A\left[T_{1}, \ldots, T_{n}\right] /\left(F_{1}(T), \ldots, F_{r}(T)\right) \rightarrow \mathcal{O}_{U, x^{\prime}}
$$

to be $\psi\left(\bar{T}_{i}\right)=t_{i}$ for all $i$. It is easy to see that

$$
\psi^{-1}\left(m_{U, x^{\prime}}\right)=\left(m_{A}, \bar{T}_{1}, \ldots, \bar{T}_{n}\right) .
$$

Thus it is sufficient to show that $\psi$ is étale. Let

$$
\mu: \widehat{A} \llbracket T_{1}, \ldots, T_{n} \rrbracket /\left(F_{1}, \ldots, F_{r}\right) \rightarrow \widehat{A} \llbracket X_{1}, \ldots, X_{n} \rrbracket /\left(F_{1}, \ldots, F_{r}\right)
$$

be a homomorphism given by the composition of homomorphisms

$$
\widehat{A} \llbracket T_{1}, \ldots, T_{n} \rrbracket /\left(F_{1}, \ldots, F_{r}\right) \stackrel{\widehat{\psi}}{\longrightarrow} \widehat{\mathcal{O}}_{U, x^{\prime}}=\widehat{\mathcal{O}}_{X, x} \stackrel{\phi^{-1}}{\longrightarrow} \widehat{A} \llbracket X_{1}, \ldots, X_{n} \rrbracket /\left(F_{1}, \ldots, F_{r}\right) .
$$

By the above claim and Lemma 1.1.1, $\mu$ is surjective. Hence, by the following Lemma 1.1.4, it must be an isomorphism. Therefore so is $\widehat{\psi}$. This means that $\psi$ is étale because $x^{\prime}$ and $\left(m_{A}, \bar{T}_{1}, \ldots, \bar{T}_{n}\right)$ have the same residue field.

Finally we consider the following lemma concerning the bijectivity of a ring homomorphism.

Lemma 1.1.4. Let $\phi: A \rightarrow A$ be an endomorphism of a noetherian ring. If $\phi$ is surjective, then $\phi$ is injective.

Proof. We set $I_{n}=\operatorname{Ker}\left(\phi^{n}\right)$ for $n \geq 1$. Since $\phi$ is surjective, we can see that $\phi\left(I_{n+1}\right)=I_{n}$ for all $n \geq 1$. Moreover there is $N \geq 1$ such that $I_{N+1}=I_{N}$ because $A$ is noetherian and $I_{n} \subseteq I_{n+1}$ for all $n \geq 1$. Therefore

$$
\operatorname{Ker}(\phi)=I_{1}=\phi^{N}\left(I_{N+1}\right)=\phi^{N}\left(I_{N}\right)=\{0\} .
$$




\section{$\S 1.2$. Semistable varieties and semistable schemes}

Let $k$ be an algebraically closed field and $X$ an algebraic scheme over $k$. A closed point $x$ of $X$ is called a semistable point of $X$ if the completion of the local ring at $x$ is isomorphic to a ring of type

$$
k \llbracket X_{1}, \ldots, X_{n} \rrbracket /\left(X_{1} \cdots X_{l}\right) .
$$

The number $l$ is called the multiplicity of $X$ at $x$, and is denoted by $\operatorname{mult}_{x}(X)$. Moreover we say $X$ is a semistable variety over $k$ if every closed point is a semistable point. By the following Proposition 1.2.1, the set of all semistable closed points of $X$ is the set of the closed points belonging to a Zariski open set. Thus we say a point $x$ of $X$ ( $x$ is not necessarily closed) is a semistable point if there is a Zariski open set $U$ of $X$ such that $x \in U$ and every closed point of $U$ is a semistable point. Note that the above definition of the semistability at $x$ (not necessarily closed) is equivalent to say that there is an étale neighborhood $U$ at $x$ which is étale over $\operatorname{Spec}\left(k\left[X_{1}, \ldots, X_{n}\right] /\left(X_{1} \cdots X_{l}\right)\right)$.

Let $\Omega$ be an algebraically closed field such that $k$ is a subfield of $\Omega$. Note that if $X$ is a semistable variety over $k$, then so is $X_{\Omega}=X \times_{\operatorname{Spec}(k)} \operatorname{Spec}(\Omega)$ over $\Omega$ (cf. Proposition 1.2.2).

Let $S$ be a locally noetherian scheme and $f: X \rightarrow S$ a morphism of finite type. First we assume that $S=\operatorname{Spec}(F)$ for some field $F$. Let $\bar{F}$ be the algebraic closure of $F, X^{\prime}=X \times_{\operatorname{Spec}(F)} \operatorname{Spec}(\bar{F})$, and $\pi: X^{\prime} \rightarrow X$ the canonical morphism. A point $x$ of $X$ is called a semistable point of $X$ if every point $x^{\prime}$ of $X^{\prime}$ with $\pi\left(x^{\prime}\right)=x$ is a semistable point. For a general $S$, we say $f: X \rightarrow S$ is semistable at $x \in X$ if $f$ is flat at $x$ and $x$ is a semistable point of the fiber $f^{-1}(f(x))$ passing through $x$. Moreover we say $X$ is a semistable scheme over $S$ if $f$ is semistable at all points of $X$. By Proposition 1.2.2, for a flat morphism $f: X \rightarrow S, X$ is a semistable scheme over $S$ if and only if, for any algebraically closed field $\Omega$, any morphism $\operatorname{Spec}(\Omega) \rightarrow S$ and any closed point $x^{\prime} \in X \times{ }_{S} \operatorname{Spec}(\Omega)$, the completion of the local ring at $x^{\prime}$ is isomorphic to a ring of type

$$
\Omega \llbracket X_{1}, \ldots, X_{n} \rrbracket /\left(X_{1} \cdots X_{l}\right) .
$$

We say a semistable scheme $X$ over $S$ is proper if $X$ is proper over $S$. Moreover a proper semistable scheme $X$ over $S$ is said to be connected if $f_{*}\left(\mathcal{O}_{X}\right)=\mathcal{O}_{S}$.

In the remaining of this subsection, let us consider elementary properties of semistable varieties.

Proposition 1.2.1. $\quad$ Let $X$ be an algebraic scheme over an algebraically closed field $k$. If $x$ is a semistable closed point of $X$, then there is a Zariski 
open set $U$ of $X$ such that $x \in U$ and every closed point of $U$ is a semistable point.

Proof. By Proposition 1.1.3, there are an étale neighborhood $\pi:\left(U, x^{\prime}\right) \rightarrow$ $(X, x)$ of $x$ and an étale morphism

$$
\rho: U \rightarrow \operatorname{Spec}\left(k\left[T_{1}, \ldots, T_{n}\right] /\left(T_{1} \cdots T_{l}\right)\right)
$$

with $\rho\left(x^{\prime}\right)=(0, \ldots, 0)$. Note that $\operatorname{Spec}\left(k\left[T_{1}, \ldots, T_{n}\right] /\left(T_{1} \cdots T_{l}\right)\right)$ is a semistable variety over $k$. Thus so is $U$ over $k$. Therefore every closed point of $\pi(U)$ is a semistable point.

Proposition 1.2.2. $\quad$ Let $X$ be an algebraic scheme over an algebraically closed field $k$. Let $\Omega$ be an algebraically closed field such that $k$ is a subfield of $\Omega$. Let $\pi: X_{\Omega}=X \times_{\operatorname{Spec}(k)} \operatorname{Spec}(\Omega) \rightarrow X$ be the canonical morphism. For $y \in X_{\Omega}$, if $x=\pi(y)$ is a semistable point, then so is $y$.

Proof. Let $U$ be an open set of $X$ containing $x$ such that every closed point of $U$ is a semistable point.

First we assume that $y$ is a closed point. Let us choose a closed point $o \in \overline{\{x\}} \cap U$. By using Proposition 1.1.3 and shrinking $U$ around $o$ if necessary, there are étale morphisms

$$
f: V \rightarrow U \text { and } g: V \rightarrow W=\operatorname{Spec}\left(k\left[X_{1}, \ldots, X_{n}\right] /\left(X_{1} \cdots X_{l}\right)\right)
$$

of algebraic schemes over $k$ and closed points $o^{\prime} \in V$ and $o^{\prime \prime} \in W$ such that $f\left(o^{\prime}\right)=o$ and $g\left(o^{\prime}\right)=o^{\prime \prime}=(0, \ldots, 0)$. Since $x \in U, o \in \overline{\{x\}} \cap U$ and $f$ is faithfully flat at $o^{\prime}$, we can find $x^{\prime} \in V$ with $f\left(x^{\prime}\right)=x$ and $o^{\prime} \in \overline{\left\{x^{\prime}\right\}}$. Here we set

$$
\left\{\begin{array}{l}
U_{\Omega}=U \times_{\operatorname{Spec}(k)} \operatorname{Spec}(\Omega), \\
V_{\Omega}=V \times_{\operatorname{Spec}(k)} \operatorname{Spec}(\Omega), \\
W_{\Omega}=\operatorname{Spec}\left(\Omega\left[X_{1}, \ldots, X_{n}\right] /\left(X_{1} \cdots X_{l}\right)\right)
\end{array}\right.
$$

and the induced morphisms $V_{\Omega} \rightarrow U_{\Omega}$ and $V_{\Omega} \rightarrow W_{\Omega}$ are denoted by $f_{\Omega}$ and $g_{\Omega}$ respectively. Then $y \in U_{\Omega}$. Let $\tilde{y}: \operatorname{Spec}(\Omega) \rightarrow U_{\Omega}$ be the morphism induced by $y$. Let $\kappa(y), \kappa(x)$ and $\kappa\left(x^{\prime}\right)$ be the residue fields of $y, x$ and $x^{\prime}$ respectively. Then there is an embedding $\iota: \kappa\left(x^{\prime}\right) \hookrightarrow \Omega$ over $k$ such that the following diagram is commutative:

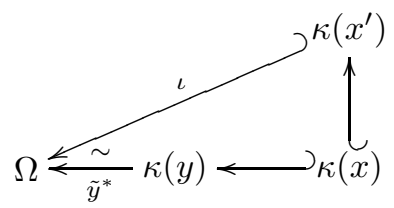


This yields a morphism $\beta: \operatorname{Spec}(\Omega) \rightarrow V_{\Omega}$ such that the diagram

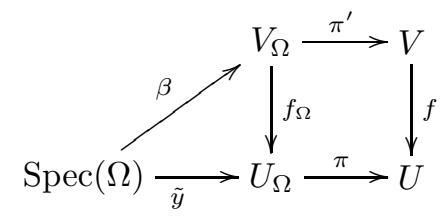

is commutative and the image of $\pi^{\prime} \circ \beta$ is $x^{\prime}$. Let $y^{\prime}$ be the image of $\beta$. Then $f_{\Omega}\left(y^{\prime}\right)=y$. Note that $f_{\Omega}$ and $g_{\Omega}$ are étale and the residue fields of $y, y^{\prime}$ and $y^{\prime \prime}=g_{\Omega}\left(y^{\prime}\right)$ are $\Omega$. Thus we can see that

$$
\widehat{\mathcal{O}}_{X_{\Omega}, y} \simeq \widehat{\mathcal{O}}_{V_{\Omega}, y^{\prime}} \simeq \widehat{\mathcal{O}}_{W_{\Omega}, y^{\prime \prime}} .
$$

We set $y^{\prime \prime}=\left(a_{1}, \ldots, a_{n}\right) \in \mathbb{A}^{n}(\Omega)$ and $I=\left\{i \mid a_{i}=0\right.$ and $\left.i=1, \ldots, l\right\}$. Note that $I \neq \emptyset$ because $y^{\prime \prime} \in W_{\Omega}$. Therefore, if we set $Z_{i}=X_{i}-a_{i}$ and $Z=\prod_{i \in I} Z_{i}$, then it is easy to see that

$$
\widehat{\mathcal{O}}_{W_{\Omega}, y^{\prime \prime}}=\Omega \llbracket Z_{1}, \ldots, Z_{n} \rrbracket /(Z) .
$$

Thus we get our lemma in the case where $y$ is a closed point.

Next we consider a general case. We set $U_{\Omega}=\pi^{-1}(U)$. Then, by the previous observation, every closed point of $U_{\Omega}$ is a semistable point. On the other hand, $y \in U_{\Omega}$. Thus $y$ is a semistable point.

\section{§2. Some Facts on Log Structures}

In this section, we consider several facts concerning log structures, which will be used later.

\section{§2.1. $\quad$ Ring extension for a good chart}

Here we consider a ring extension to get a good chart. This is a partial result of a proposition in the unpublished Ogus' paper [8].

Proposition 2.1.1. $\quad$ Let $(A, m)$ be a noetherian local ring, $S=\operatorname{Spec}(A)$ and $s$ the closed point of $S$. Let $M_{S}$ be a fine log structure on $S$. Then there is a local homomorphism $f:(A, m) \rightarrow(B, n)$ of noetherian local rings with the following properties:

(1) $B / n$ is algebraic over $A / m$, and $f$ is flat and quasi-finite. 
(2) Let $f^{a}: S^{\prime}=\operatorname{Spec}(B) \rightarrow S=\operatorname{Spec}(A)$ be the induced morphism, $s^{\prime}$ the closed point of $S^{\prime}=\operatorname{Spec}(B)$, and $M_{S^{\prime}}=\left(f^{a}\right)^{*}\left(M_{S}\right)$. There are a fine and sharp monoid $Q$ and a homomorphism $\pi_{Q}: Q \rightarrow M_{S^{\prime}, s^{\prime}}$ such that $Q \rightarrow M_{S^{\prime}, \bar{s}^{\prime}} \rightarrow \bar{M}_{S^{\prime}, \bar{s}^{\prime}}$ is bijective.

Proof. Let us begin with the following lemma:

Lemma 2.1.2. Let $G$ be a finitely generated abelian group and $R$ a ring. Let us fix an element $\delta$ of $\operatorname{Ext}^{1}\left(G, R^{\times}\right)$. Then there are $u_{1}, \ldots, u_{l} \in R^{\times}$and integers $a_{1}, \ldots, a_{l} \geq 2$ with the following property:

(1) The product $a_{1} \cdots a_{l}$ of integers $a_{1}, \ldots, a_{l}$ is equal to the order of the torsion part of $G$.

(2) For any homomorphism $f: R \rightarrow S$ of rings, if there are $v_{1}, \ldots, v_{l} \in S$ with $v_{i}^{a_{i}}=f\left(u_{i}\right)$ for all $i$, then the image of $\delta$ via the canonical homomorphism

$$
\operatorname{Ext}^{1}\left(G, R^{\times}\right) \rightarrow \operatorname{Ext}^{1}\left(G, S^{\times}\right)
$$

is zero.

Proof. By the fundamental theorem of abelian groups, we have the following exact sequence:

$$
0 \longrightarrow \mathbb{Z}^{l} \stackrel{\phi}{\longrightarrow} \mathbb{Z}^{l^{\prime}} \longrightarrow G \longrightarrow 0,
$$

where $\phi$ is given by $\phi\left(x_{1}, \ldots, x_{l}\right)=\left(a_{1} x_{1}, \ldots, a_{l} x_{l}, 0, \ldots, 0\right)$ for some integers $a_{1}, \ldots, a_{l} \geq 2$. Note that $a_{1} \cdots a_{l}$ is equal to the order of the torsion part of $G$. The above exact sequence yields an exact sequence

$$
\operatorname{Hom}\left(\mathbb{Z}^{l^{\prime}}, R^{\times}\right) \stackrel{\phi_{R}^{*}}{\longrightarrow} \operatorname{Hom}\left(\mathbb{Z}^{l}, R^{\times}\right) \stackrel{\alpha_{R}}{\longrightarrow} \operatorname{Ext}^{1}\left(G, R^{\times}\right) \longrightarrow \operatorname{Ext}^{1}\left(\mathbb{Z}^{l^{\prime}}, R^{\times}\right) .
$$

Note that $\operatorname{Ext}^{1}\left(\mathbb{Z}^{l^{\prime}}, R^{\times}\right)=\{0\}$. Thus there is $h \in \operatorname{Hom}\left(\mathbb{Z}^{l}, R^{\times}\right)$with $\alpha_{R}(h)=$ $\delta$. We set $u_{i}=h\left(e_{i}\right)$ for $i=1, \ldots, l$, where $\left\{e_{1}, \ldots, e_{l}\right\}$ is the standard basis of $\mathbb{Z}^{l}$.

Let $f: R \rightarrow S$ be any homomorphism of rings with $v_{i}^{a_{i}}=f\left(u_{i}\right)(i=$ $1, \ldots, l)$ for some $v_{1}, \ldots, v_{l} \in S$. Let us consider the following commutative diagram:

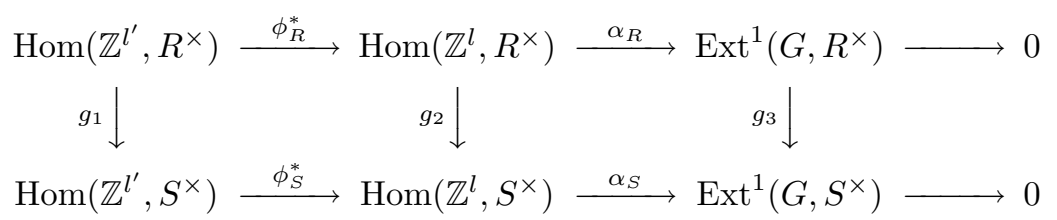


Note that $g_{2}(h)\left(e_{i}\right)=f\left(u_{i}\right)$ for $i=1, \ldots, l$. Thus, if we set $h^{\prime} \in \operatorname{Hom}\left(\mathbb{Z}^{l^{\prime}}, S^{\times}\right)$ by

$$
h^{\prime}\left(e_{i}\right)= \begin{cases}v_{i} & \text { if } i=1, \ldots, l \\ 0 & \text { if } i>l\end{cases}
$$

then $\phi_{S}^{*}\left(h^{\prime}\right)=g_{2}(h)$. Therefore

$$
g_{3}(\delta)=g_{3}\left(\alpha_{R}(h)\right)=\alpha_{S}\left(g_{2}(h)\right)=\alpha_{S}\left(\phi_{S}^{*}\left(h^{\prime}\right)\right)=0 .
$$

Let us start the proof of Proposition 2.1.1. Let $\delta \in \operatorname{Ext}^{1}\left(\bar{M}_{S, \bar{s}}^{g r}, \mathcal{O}_{S, \bar{s}}^{\times}\right)$be the extension class of

$$
0 \rightarrow \mathcal{O}_{S, \bar{s}}^{\times} \rightarrow M_{S, \bar{s}}^{g r} \rightarrow \bar{M}_{S, \bar{s}}^{g r} \rightarrow 0 .
$$

Then, by Lemma 2.1.2, there are $u_{1}, \ldots, u_{l} \in \mathcal{O}_{S, \bar{s}}^{\times}$and integers $a_{1}, \ldots, a_{l}$ with the properties as in Lemma 2.1.2. Let us choose an étale neighborhood $(U, u)$ of $s$ such that $u_{1}, \ldots, u_{l} \in \mathcal{O}_{U, u}^{\times}$. Let $B$ be the localization of

$$
\mathcal{O}_{U, u}\left[X_{1}, \ldots, X_{l}\right] /\left(X_{1}^{a_{1}}-u_{1}, \ldots, X_{l}^{a_{l}}-u_{l}\right) .
$$

at a closed point over $u$. Then $B$ is flat and quasi-finite over $A$. Let $v_{i}$ be the class of $X_{i}$ in $B$. Note that $v_{i}^{a_{i}}=u_{i}$ in $B$ for all $i$. Let $s^{\prime}$ be the closed point of $S^{\prime}=\operatorname{Spec}(B), \pi: S^{\prime} \rightarrow S$ the canonical morphism, and $M_{S^{\prime}}=\pi^{*}\left(M_{S}\right)$. Then we have an exact sequence

$$
0 \rightarrow \mathcal{O}_{S^{\prime}, \bar{s}^{\prime}}^{\times} \rightarrow M_{S^{\prime}, \bar{s}^{\prime}}^{g r} \rightarrow \bar{M}_{S^{\prime}, \bar{s}^{\prime}}^{g r} \rightarrow 0 .
$$

Since $M_{S^{\prime}, \bar{s}^{\prime}}^{g r}$ is the push-out $\mathcal{O}_{S^{\prime}, \bar{s}^{\prime}}^{\times} \bar{\otimes}_{\mathcal{O}_{S, \bar{s}}^{\times}} M_{S, \bar{s}}^{g r}$ (cf. Conventions and terminology 7.), we can see that $\bar{M}_{S^{\prime}, \bar{s}^{\prime}}^{g r}=\bar{M}_{S, \bar{s}}^{g r}$ and the extension class $\delta^{\prime}$ of the above exact sequence is the image of $\delta$ by the canonical homomorphism $\operatorname{Ext}^{1}\left(\bar{M}_{S, \bar{s}}^{g r}, \mathcal{O}_{S, \bar{s}}^{\times}\right) \rightarrow \operatorname{Ext}^{1}\left(\bar{M}_{S^{\prime}, \bar{s}^{\prime}}^{g r}, \mathcal{O}_{S^{\prime}, \bar{s}^{\prime}}^{\times}\right)$. Thus, by Lemma 2.1.2, $\delta^{\prime}=0$. Therefore we have a splitting $s: \bar{M}_{S^{\prime}, \bar{s}^{\prime}}^{g r} \rightarrow M_{S^{\prime}, \bar{s}^{\prime}}^{g r}$ of $M_{S^{\prime}, \bar{s}^{\prime}}^{g r} \rightarrow \bar{M}_{S^{\prime}, \bar{s}^{\prime}}^{g r}$. Here we set $Q=\bar{M}_{S^{\prime}, \bar{s}^{\prime}}$. Let us see that $s(q) \in M_{S^{\prime}, \bar{s}^{\prime}}$ for all $q \in Q$. Indeed, if we denote $M_{S^{\prime}, \bar{s}^{\prime}}^{g r} \rightarrow \bar{M}_{S^{\prime}, \bar{s}^{\prime}}^{g r}$ by $\pi$, then $\pi(s(q))=q$. Thus there are $u \in \mathcal{O}_{S^{\prime}, \bar{s}^{\prime}}^{\times}$ and $m \in M_{S^{\prime}, \bar{s}^{\prime}}$ with $s(q)=m \cdot u$, which implies $s(q) \in M_{S^{\prime}, \bar{s}^{\prime}}$. Moreover $Q \rightarrow M_{S^{\prime}, \bar{s}^{\prime}} \rightarrow \bar{M}_{S^{\prime}, \bar{s}^{\prime}}$ is the identity map. Further, changing $S^{\prime}$ by an étale neighborhood of $S^{\prime}$, we may assume that $Q \rightarrow M_{S^{\prime}, \bar{s}}$ is defined on $S^{\prime}$. 


\section{§2.2. The support of log structures}

In this subsection, we consider the support of log structures. The main result of this subsection is the following proposition:

Proposition 2.2.1. Let $X$ be a scheme and let $M$ and $N$ be fine log structures on $X$. Let $h: N \rightarrow M$ be a homomorphism of log structures, i.e., a homomorphism of sheaves of monoids with the following diagram commutative:

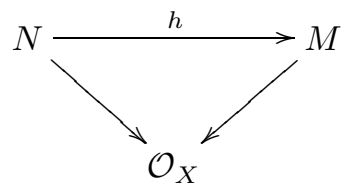

Then the set $\left\{x \in X \mid h_{\bar{x}}: N_{\bar{x}} \rightarrow M_{\bar{x}}\right.$ is surjective $\}$ is open.

Proof. It is sufficient to show that if $h_{\bar{x}}: N_{\bar{x}} \rightarrow M_{\bar{x}}$ is surjective, then there is an étale neighborhood $U$ of $x$ such that, for all $y \in U, h_{\bar{y}}: N_{\bar{y}} \rightarrow M_{\bar{y}}$ is surjective.

Claim 2.2.1.1. For $z \in X, h_{\bar{z}}: N_{\bar{z}} \rightarrow M_{\bar{z}}$ is surjective if and only if $\bar{h}_{\bar{z}}: \bar{N}_{\bar{z}} \rightarrow \bar{M}_{\bar{z}}$ is surjective.

Clearly, if $h_{\bar{z}}: N_{\bar{z}} \rightarrow M_{\bar{z}}$ is surjective, then so is $\bar{h}_{\bar{z}}: \bar{N}_{\bar{z}} \rightarrow \bar{M}_{\bar{z}}$. Conversely we assume that $\bar{h}_{\bar{z}}: \bar{N}_{\bar{z}} \rightarrow \bar{M}_{\bar{z}}$ is surjective. Let $m$ be an element of $M_{\bar{z}}$. Then there is $n \in N_{\bar{z}}$ such that $m \equiv h_{\bar{z}}(n) \bmod \mathcal{O}_{X, \bar{z}}^{\times}$, i.e., $m=u h_{\bar{z}}(n)$ for some $u \in \mathcal{O}_{X, \bar{z}}^{\times}$. Thus $m=u h_{\bar{z}}(n)=h_{\bar{z}}(u n)$.

By virtue of $[4,(2.9)]$, for a suitable étale neighborhood $U$ of $x$, there are finitely generated monoids $P$ and $Q$ together with homomorphisms $\pi: P \rightarrow$ $\left.M\right|_{U}, \mu:\left.Q \rightarrow N\right|_{U}$ and $f: Q \rightarrow P$ such that $\pi$ and $\mu$ give rise to local charts of $M$ and $N$ respectively and the diagram

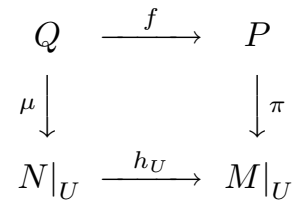

is commutative. It is easy to see that $\pi_{\bar{x}}^{-1}\left(\mathcal{O}_{X, \bar{x}}^{\times}\right)$is a finitely generated submonoid of $P$. Let $p_{1}, \ldots, p_{n}$ be generators of $\pi_{\bar{x}}^{-1}\left(\mathcal{O}_{X, \bar{x}}^{\times}\right)$. Shrinking $U$ if necessary, we may assume that

$$
\pi\left(p_{1}\right), \ldots, \pi\left(p_{n}\right) \in \mathcal{O}_{X, \bar{y}}^{\times}
$$


for all $y \in U$. Let us check that $h_{\bar{y}}: N_{\bar{y}} \rightarrow M_{\bar{y}}$ is surjective for all $y \in U$, which is equivalent to show that $\bar{h}_{\bar{y}}: \bar{N}_{\bar{y}} \rightarrow \bar{M}_{\bar{y}}$ is surjective by Claim 2.2.1.1. Note that the commutative diagram

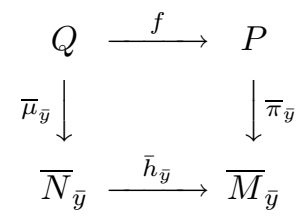

gives rise to the commutative diagram

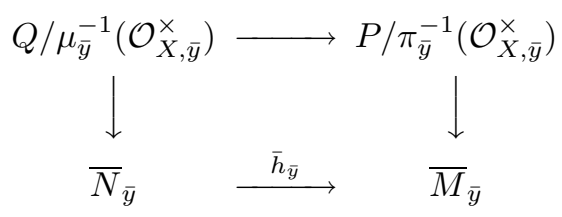

such that the vertical homomorphisms are bijective (cf. [4] and [6]). Therefore it is sufficient to see that

$$
Q / \mu_{\bar{y}}^{-1}\left(\mathcal{O}_{X, \bar{y}}^{\times}\right) \rightarrow P / \pi_{\bar{y}}^{-1}\left(\mathcal{O}_{X, \bar{y}}^{\times}\right)
$$

is surjective, which is equivalent to say that

$$
Q \rightarrow P / \pi_{\bar{y}}^{-1}\left(\mathcal{O}_{X, \bar{y}}^{\times}\right)
$$

is surjective. On the other hand, since $\pi_{\bar{x}}^{-1}\left(\mathcal{O}_{X, \bar{x}}^{\times}\right) \subseteq \pi_{\bar{y}}^{-1}\left(\mathcal{O}_{X, \bar{y}}^{\times}\right)$, it suffices to show that

$$
Q \rightarrow P / \pi_{\bar{x}}^{-1}\left(\mathcal{O}_{X, \bar{x}}^{\times}\right)
$$

is surjective, which is nothing more than the surjectivity of $\bar{h}_{\bar{x}}: \bar{N}_{\bar{x}} \rightarrow \bar{M}_{\bar{x}}$.

Corollary 2.2.2. $\quad$ Let $X$ be a scheme and $M$ a fine log structure on $X$. Then the set $\operatorname{Supp}(M)=\left\{x \in X \mid M_{\bar{x}}\right.$ is not trivial $\}$ is closed.

Proof. There is a natural homomorphism $\mathcal{O}_{X}^{\times} \rightarrow M$. Thus this is a consequence of the above proposition.

Corollary 2.2.3. $\quad$ Let $X$ and $Y$ be schemes and let $M$ and $N$ be fine log structures on $X$ and $Y$ respectively. Let $(f, h):(X, M) \rightarrow(Y, N)$ be a log morphism. 
(1) The set

$$
\operatorname{Supp}(M / N)=\left\{x \in X \mid N_{\overline{f(x)}} \times \mathcal{O}_{X, \bar{x}}^{\times} \rightarrow M_{\bar{x}} \text { is not surjective }\right\}
$$

is closed.

(2) Let $\rho: Y^{\prime} \rightarrow Y$ be a morphism of schemes and $X^{\prime}=X \times_{Y} Y^{\prime}$. We set the induced morphisms as follows:

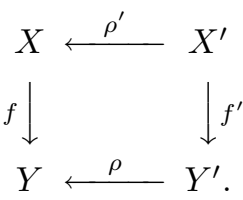

Then $\rho^{\prime-1}(\operatorname{Supp}(M / N))=\operatorname{Supp}\left(\rho^{\prime *}(M) / \rho^{*}(N)\right)$.

Proof. (1) Note that the surjectivity of $N_{\overline{f(x)}} \times \mathcal{O}_{X, \bar{x}}^{\times} \rightarrow M_{\bar{x}}$ is equivalent to the surjectivity of $f^{*}(N)_{\bar{x}} \rightarrow M_{\bar{x}}$. Thus it follows from Proposition 2.2.1.

(2) For $x^{\prime} \in X^{\prime}$, we set $x=\rho^{\prime}\left(x^{\prime}\right)$. Note that $\rho^{\prime *}\left(f^{*}(N)\right)=f^{\prime *}\left(\rho^{*}(N)\right)$. Thus we have a commutative diagram:

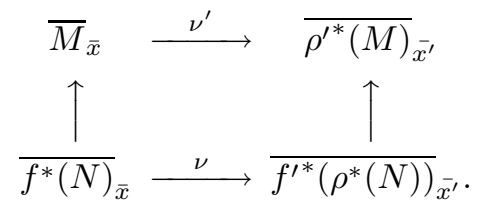

Note that the horizontal homomorphisms $\nu^{\prime}$ and $\nu$ are bijective. Hence, by using Claim 2.2.1.1 of Proposition 2.2.1, we have (2).

\section{$\S 3 . \quad$ Local Structure Theorem}

In this section, we consider the following fundamental structure theorem of this paper.

Theorem 3.1 (Local structure theorem). Let $(f, h):\left(X, M_{X}\right) \rightarrow\left(S, M_{S}\right)$ be a smooth and integral morphism of fine log schemes. Let $x$ be a point of $X$ and $s=f(x)$. We assume that $f: X \rightarrow S$ is semistable at $x$. Then we have the following:

(1) If $f$ is smooth at $x$, then there is a submonoid $N$ of $\bar{M}_{X, \bar{x}}$ such that $\bar{M}_{X, \bar{x}}=$ $\bar{h}_{\bar{x}}\left(\bar{M}_{S, \bar{s}}\right) \times N$ and $N$ is isomorphic to $\mathbb{N}^{a}$ for some non-negative integer a. Moreover every element of $N$ is regular (For the definition of regularity, see Conventions and terminology 5). 
(2) If $f$ is not smooth at $x$ and $\bar{h}_{\bar{x}}: \bar{M}_{S, \bar{s}} \rightarrow \bar{M}_{X, \bar{x}}$ splits, there is a submonoid $N$ of $\bar{M}_{X, \bar{x}}$ such that $\bar{M}_{X, \bar{x}}=\bar{h}_{\bar{x}}\left(\bar{M}_{S, \bar{s}}\right) \times N$ and $N$ is isomorphic to the monoid arising from monic monomials of

$$
\mathbb{Z}\left[U_{1}, U_{2}, \ldots, U_{a}\right] /\left(U_{1}^{2}-U_{2}^{2}\right)
$$

for some $a \geq 2$. In this case, the characteristic of the residue field of $\mathcal{O}_{X, \bar{x}}$ is not equal to 2, and every element of $N$ is regular.

(3) If $f$ is not smooth at $x$ and $\bar{h}_{\bar{x}}: \bar{M}_{S, \bar{s}} \rightarrow \bar{M}_{X, \bar{x}}$ does not split, then $\bar{M}_{X, \bar{x}}$ has a semistable structure $\left(\sigma, q_{0}, \Delta, B\right)$ over $\bar{M}_{S, \bar{s}}$ for some $\sigma \subseteq \bar{M}_{X, \bar{x}}$ with $\#(\sigma) \geq 2, q_{0} \in \bar{M}_{S, \bar{s}}$ and $\Delta, B \in \mathbb{N}^{\sigma}$ (For the definition of semistable structure, see Conventions and terminology 10). More precisely, $\sigma$ is the set of all irreducible elements of $\bar{M}_{X, \bar{x}}$ not lying in $\bar{h}_{\bar{x}}\left(\bar{M}_{S, \bar{s}}\right)$. Further every element of $\sigma \backslash \operatorname{Supp}(\Delta)$ is regular.

Proof. First we will prove this theorem except the regularity of elements of either $N$ or $\sigma \backslash \operatorname{Supp}(\Delta)$ in each case (1), (2), (3).

Let us consider the geometric fiber $X_{\bar{s}}=X \times_{S} \operatorname{Spec}(\overline{\kappa(s)})$ over $s$. Note that $\bar{M}_{X, \bar{x}}$ is canonically isomorphic to $\bar{M}_{X_{\bar{s}}, \bar{x}}$, where $M_{X_{\bar{s}}}=\left.M_{X}\right|_{X_{\bar{s}}}$. Thus we may assume that $S=\operatorname{Spec}(k)$ for some algebraically closed field $k$. Hence the theorem follows from [6, Theorem 3.1], provided we show that, in the case (2), $N$ is isomorphic to the monoid $T$ arising from monic monomials of

$$
\mathbb{Z}\left[U_{1}, U_{2}, \ldots, U_{a}\right] /\left(U_{1}^{2}-U_{2}^{2}\right) .
$$

Let $T_{k}$ be the monoid arising from monic monomials of

$$
k\left[U_{1}, U_{2}, \ldots, U_{a}\right] /\left(U_{1}^{2}-U_{2}^{2}\right) .
$$

We need to show the natural homomorphism $T \rightarrow T_{k}$ is bijective. Let $\bar{U}_{1}^{e_{1}} \bar{U}_{2}^{e_{2}}$ $\cdots \bar{U}_{a}^{e_{a}}$ and $\bar{U}_{1}^{e_{1}^{\prime}} \bar{U}_{2}^{e_{2}^{\prime}} \cdots \bar{U}_{a}^{e_{a}^{\prime}}$ be elements of $T$. Clearly we may assume that $e_{1}, e_{1}^{\prime} \in\{0,1\}$. We suppose that $\bar{U}_{1}^{e_{1}} \bar{U}_{2}^{e_{2}} \cdots \bar{U}_{a}^{e_{a}}=\bar{U}_{1}^{e_{1}^{\prime}} \bar{U}_{2}^{e_{2}^{\prime}} \cdots \bar{U}_{a}^{e_{a}^{\prime}}$ in $k\left[U_{1}, U_{2}\right.$, $\left.\ldots, U_{a}\right] /\left(U_{1}^{2}-U_{2}^{2}\right)$. Then there is $\phi \in k\left[U_{1}, \ldots U_{a}\right]$ with

$$
U_{1}^{e_{1}} U_{2}^{e_{2}} \cdots U_{a}^{e_{a}}-U_{1}^{e_{1}^{\prime}} U_{2}^{e_{2}^{\prime}} \cdots U_{a}^{e_{a}^{\prime}}=\left(U_{1}^{2}-U_{2}^{2}\right) \phi .
$$

Comparing the degrees with respect to $U_{1}$ of both sides, we can see that $\phi=0$. Therefore $\left(e_{1}, \ldots, e_{a}\right)=\left(e_{1}^{\prime}, \ldots, e_{a}^{\prime}\right)$.

The rest is to prove that elements of either $N$ or $\sigma \backslash \operatorname{Supp}(\Delta)$ are regular in each case (1), (2), (3). 
Let $\left(\mathcal{O}_{S, s}, m_{S, s}\right) \rightarrow(A, m)$ be a flat local homomorphism of local rings. We set $S^{\prime}=\operatorname{Spec}(A), X^{\prime}=X \times{ }_{S} S^{\prime}$ and the induced morphisms as follows:

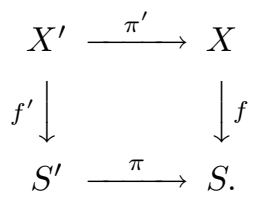

Let us choose $x^{\prime} \in X^{\prime}$ with $f^{\prime}\left(x^{\prime}\right)=m$ and $\pi^{\prime}\left(x^{\prime}\right)=x$. Then, since $\mathcal{O}_{X, x} \rightarrow$ $\mathcal{O}_{X^{\prime}, x^{\prime}}$ is faithfully flat, if regularity holds at $x^{\prime}$, then so does at $x$.

Let $k$ be the algebraic closure of the residue field at $x$. Note that by virtue of [3, EGA III, Chapter 0, 10.3.1], there are a noetherian local ring $(A, m)$ and a local homomorphism $\left(\mathcal{O}_{S, s}, m_{S, s}\right) \rightarrow(A, m)$ such that $m_{S, s} A=m, A / m$ is isomorphic to $k$ over $\mathcal{O}_{S, s} / m_{S, s}$ and that $A$ is flat over $\mathcal{O}_{S, s}$. Therefore we may assume that $\mathcal{O}_{S, s} / m_{S, s}$ is algebraically closed and $x$ is a closed point. Moreover, by using Proposition 2.1.1, we may further assume that there are a fine and sharp monoid $Q$ and a homomorphism $\pi_{Q}: Q \rightarrow M_{S, s}$ such that $Q \rightarrow M_{S, \bar{s}} \rightarrow \bar{M}_{S, \bar{s}}$ is bijective. Hence, by [6] or Ogus' paper [8], there is a fine and sharp monoid $P$ together with homomorphisms $f: Q \rightarrow P$ and $\pi_{P}: P \rightarrow M_{X, \bar{x}}$ such that the following properties are satisfied:

(a) The diagram

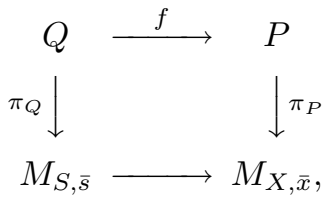

is commutative.

(b) The induced homomorphism $P \rightarrow M_{X, \bar{x}} \rightarrow \bar{M}_{X, \bar{x}}$ is bijective.

(c) The natural homomorphism

$$
\mathcal{O}_{S, \bar{s}} \otimes_{\mathcal{O}_{S, \bar{s}}[Q]} \mathcal{O}_{S, \bar{s}}[P] \rightarrow \mathcal{O}_{X, \bar{x}}
$$

is flat.

Since $\mathcal{O}_{S, \bar{s}} \otimes_{\mathcal{O}_{S, \bar{s}}[Q]} \mathcal{O}_{S, \bar{s}}[P] \rightarrow \mathcal{O}_{X, \bar{x}}$ is flat, it is sufficient to see the regularity of each element in $\mathcal{O}_{S, \bar{s}} \otimes_{\mathcal{O}_{S, \bar{s}}[Q]} \mathcal{O}_{S, \bar{s}}[P]$.

If there is a submonoid $N$ of $P$ with $P=f(Q) \times N$, then

$$
\mathcal{O}_{S, \bar{s}} \otimes_{\mathcal{O}_{S, \bar{s}}[Q]} \mathcal{O}_{S, \bar{s}}[P]=\mathcal{O}_{S, \bar{s}}[N]
$$


Thus, for the case (1), the regularity of $N$ is obvious because $N$ is free. For the case (2), the assertions follow from Lemma 3.2 below.

Next we assume that $f: Q \rightarrow P$ does not split. Let us set $\sigma=\left\{p_{1}, \ldots, p_{r}\right\}$ such that $\operatorname{Supp}(\Delta)=\left\{p_{1}, \ldots, p_{l}\right\}$. Moreover we set $x_{i}=\alpha\left(\pi_{P}\left(p_{i}\right)\right)$ and $t=$ $\beta\left(\pi_{Q}\left(q_{0}\right)\right)$, where $\alpha: M_{X} \rightarrow \mathcal{O}_{X}$ and $\beta: M_{S, \bar{s}} \rightarrow \mathcal{O}_{S, \bar{s}}$ are the canonical homomorphisms. Then

$$
\mathcal{O}_{S, \bar{s}} \otimes_{\mathcal{O}_{S, \bar{s}}[Q]} \mathcal{O}_{S, \bar{s}}[P]=\mathcal{O}_{S, \bar{s}}\left[X_{1}, \ldots, X_{r}\right] /\left(X_{1} \cdots X_{l}-t X_{l+1}^{b_{l+1}} \cdots X_{r}^{b_{r}}\right),
$$

where $b_{i}=B\left(p_{i}\right)$ and $x_{i}$ is the class of $X_{i}$. Thus the assertions follow from Lemma 3.2 below.

Lemma 3.2. Let $A$ be a ring. Then we have the following:

(1) Let $A[X]$ be the polynomial ring of one variable over $A$. For a regular element $a \in A, X$ is regular in $A[X] /\left(X^{2}-a\right)$, that is, the multiplication of $X$ in $A[X] /\left(X^{2}-a\right)$ is injective.

(2) Let $A\left[X_{1}, \ldots, X_{l}\right]$ be the polynomial ring of $l$-variables over $A$. For $a \in A$, let us consider a ring $R$ given by $R=A\left[X_{1}, \ldots, X_{l}\right] /\left(X_{1} \cdots X_{l}-a\right)$. If $\alpha$ is a regular element of $A$, then so is $\alpha$ in $R$.

Proof. (1) We assume that $X f(X)=\left(X^{2}-a\right) g(X)$ for some $f(X), g(X) \in$ $A[X]$. We set $g(X)=X h(X)+c$ for some $h(X) \in A[X]$ and $c \in A$. Then

$$
c a=X\left(h(X)\left(X^{2}-a\right)+c X-f(X)\right) .
$$

Thus, $c a=0$. Since $a$ is regular, $c$ must be zero. Therefore

$$
X f(X)=X\left(X^{2}-a\right) h(X),
$$

which implies $f(X)=\left(X^{2}-a\right) h(X)$ because $X$ is regular in $A[X]$.

(2) It is sufficient to show that $R$ is a free $A$-module. We set

$$
M=\left\{\sum_{(1, \ldots, 1) \not \subset I} a_{I} X^{I} \in A\left[X_{1}, \ldots, X_{n}\right]\right\} .
$$

Then it is easy to see that the natural $A$-module homomorphism $M \rightarrow R$ is bijective. Thus $R$ is a free $A$-module.

Remark 3.3. The semistable structure of $\bar{h}_{\bar{x}}: \bar{M}_{S, \bar{s}} \rightarrow \bar{M}_{X, \bar{x}}$ in the case (3) of Theorem 3.1 is uniquely determined by virtue of a result in [7], which is not needed in this paper. 


\section{$\S 4 . \quad$ Rigidity Theorem}

First of all, we would like to define the admissibility of morphisms. Let $k$ be an algebraically closed field, and let $\phi: X \rightarrow Y$ be a morphism of algebraic schemes over $k$. Let $Z$ be a subscheme of $Y$. We say $\phi$ is admissible with respect to $Z$ if, for any irreducible component $X^{\prime}$ of $X, \phi\left(X^{\prime}\right) \not \subset Z$.

Let $f: X \rightarrow S$ and $g: Y \rightarrow S$ be schemes of finite type over a locally noetherian scheme $S$, and let $M_{Y}$ and $M_{S}$ be fine $\log$ structures of $Y$ and $S$ such that $g$ extends to a $\log$ morphism $\left(Y, M_{Y}\right) \rightarrow\left(S, M_{S}\right)$. As in Corollary 2.2.3, the closed set $\operatorname{Supp}\left(M_{Y} / M_{S}\right)$ is given by

$$
\left\{y \in Y \mid M_{S, \overline{g(y)}} \times \mathcal{O}_{Y, \bar{y}}^{\times} \rightarrow M_{Y, \bar{y}} \text { is not surjective }\right\} .
$$

Let $\phi: X \rightarrow Y$ be a morphism over $S$. For a point $s \in S$, we say $\phi: X \rightarrow Y$ is admissible over $s$ with respect to $M_{Y} / M_{S}$, if

$$
\phi \times_{S} \operatorname{id}_{\operatorname{Spec}(\overline{\kappa(s)})}: X \times_{S} \operatorname{Spec}(\overline{\kappa(s)}) \rightarrow Y \times_{S} \operatorname{Spec}(\overline{\kappa(s)})
$$

is admissible with respect to $\left.\operatorname{Supp}\left(M_{Y} / M_{S}\right)\right|_{Y \times_{S} \operatorname{Spec}(\overline{\kappa(s)})}$. If $\phi: X \rightarrow Y$ is admissible over any points of $S$ with respect to $M_{Y} / M_{S}$, then $\phi$ is said to be admissible with respect to $M_{Y} / M_{S}$. By (2) of Corollary $2.2 .3, \phi$ is admissible over $s$ with respect to $M_{Y} / M_{S}$ if and only if

$$
\phi \times_{S} \operatorname{id}_{\operatorname{Spec}(\overline{\kappa(s)})}: X \times_{S} \operatorname{Spec}(\overline{\kappa(s)}) \rightarrow Y \times_{S} \operatorname{Spec}(\overline{\kappa(s)})
$$

is admissible with respect to $\left(\left.M_{Y}\right|_{Y \times_{S} \operatorname{Spec}(\overline{\kappa(s)})}\right) /\left(\left.M_{S}\right|_{\operatorname{Spec}(\overline{\kappa(s)})}\right)$.

The following theorem is the main theorem of this paper.

Theorem 4.1. Let $X, Y$ and $S$ be locally noetherian schemes, and let $M_{X}, M_{Y}$ and $M_{S}$ be fine log structures of $X, Y$ and $S$ respectively. Let $\left(X, M_{X}\right) \rightarrow\left(S, M_{S}\right)$ and $\left(Y, M_{Y}\right) \rightarrow\left(S, M_{S}\right)$ be integral and log smooth morphisms, and let $\phi: X \rightarrow Y$ be a morphism over $S$. Let us fix a point $s \in S$. We assume that $X \rightarrow S$ and $Y \rightarrow S$ are semistable at any points lying over $s$ and that $\phi: X \rightarrow Y$ is admissible over $s$ with respect to $M_{Y} / M_{S}$. If

$$
(\phi, h):\left(X, M_{X}\right) \rightarrow\left(Y, M_{Y}\right) \quad \text { and } \quad\left(\phi, h^{\prime}\right):\left(X, M_{X}\right) \rightarrow\left(Y, M_{Y}\right)
$$

are extensions of $\phi: X \rightarrow Y$ as log morphisms over $\left(S, M_{S}\right)$, then, for all closed points $x$ lying over $s, h_{\bar{x}}=h_{\bar{x}}^{\prime}$ as homomorphisms $M_{Y, \overline{\phi(x)}} \rightarrow M_{X, \bar{x}}$ of the stalks of étale topology.

Proof. Since this is a local problem, we may assume that $S=\operatorname{Spec}(A)$ for a noetherian local ring $(A, m)$. Let $\rho:(A, m) \rightarrow(B, n)$ be a local homomorphism of local rings such that $B / n$ is algebraic over $A / m$. We denote the closed 
point of $S$ by $s$ and the closed point of $S^{\prime}=\operatorname{Spec}(B)$ by $s^{\prime}$. We set $X^{\prime}=X \times_{S} S^{\prime}$, $Y^{\prime}=Y \times_{S} S^{\prime}, M_{X^{\prime}}=\pi_{X}^{*}\left(M_{X}\right), M_{Y^{\prime}}=\pi_{Y}^{*}\left(M_{Y}\right)$, and $M_{S^{\prime}}=\pi_{S}^{*}\left(M_{S}\right)$, where $\pi_{X}: X^{\prime} \rightarrow X, \pi_{Y}: Y^{\prime} \rightarrow Y$ and $\pi_{S}: S^{\prime} \rightarrow S$ are the canonical morphisms. Let $\phi_{S^{\prime}}: X^{\prime} \rightarrow Y^{\prime}$ be the morphism given by $\phi_{S^{\prime}}=\phi \times_{S} \operatorname{id}_{S^{\prime}}$.

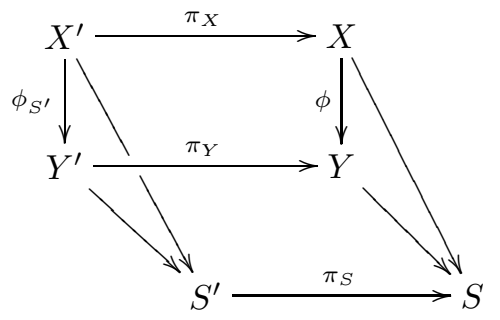

Then we have log morphisms

$$
\left(\phi_{S^{\prime}}, h_{S^{\prime}}\right),\left(\phi_{S^{\prime}}, h_{S^{\prime}}^{\prime}\right):\left(X^{\prime}, M_{X^{\prime}}\right) \rightarrow\left(Y^{\prime}, M_{Y^{\prime}}\right)
$$

over $\left(S^{\prime}, M_{S^{\prime}}\right)$, where $h_{S^{\prime}}$ and $h_{S^{\prime}}^{\prime}$ are the homomorphisms induced by $h$ and $h^{\prime}$ respectively.

Claim 4.1.1. If $\rho$ is flat and $h_{S^{\prime}, \bar{x}^{\prime}}=h_{S^{\prime}, \bar{x}^{\prime}}^{\prime}$ for all closed points $x^{\prime}$ lying over $s^{\prime}$, then $h_{\bar{x}}=h_{\bar{x}}^{\prime}$ for all closed points $x$ lying over $s$.

Let us choose a closed point $x \in X$ over $s$. Then there is a closed point $x^{\prime} \in X^{\prime}$ such that $\pi_{X}\left(x^{\prime}\right)=x$ and $x^{\prime}$ is lying over $s^{\prime}$. If we set $y=\phi(x)$ and $y^{\prime}=\phi_{S^{\prime}}\left(x^{\prime}\right)$, then $\pi_{Y}\left(y^{\prime}\right)=y$. Here we consider the natural commutative diagram:

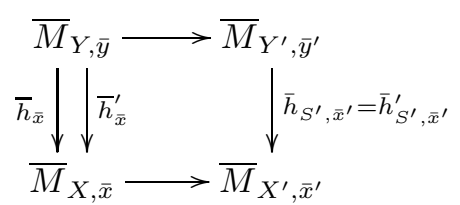

Note that $\bar{M}_{Y, \bar{y}} \rightarrow \bar{M}_{Y^{\prime}, \bar{y}^{\prime}}$ and $\bar{M}_{X, \bar{x}} \rightarrow \bar{M}_{X^{\prime}, \bar{x}^{\prime}}$ are bijective. Thus we can see that $\bar{h}_{\bar{x}}=\bar{h}_{\bar{x}}^{\prime}$. Let us pick up $w \in M_{Y, \bar{y}}$. Then, since $\bar{h}_{\bar{x}}=\bar{h}_{\bar{x}}^{\prime}$, there is $u \in \mathcal{O}_{X, \bar{x}}^{\times}$with $h_{\bar{x}}(w)=h_{\bar{x}}^{\prime}(w) \cdot u$. Here $h_{S^{\prime}, \bar{x}^{\prime}}=h_{S^{\prime}, \bar{x}^{\prime}}^{\prime}$. Thus $u$ must be 1 in $\mathcal{O}_{X^{\prime}, \bar{x}^{\prime}}$. Note that $\mathcal{O}_{X^{\prime}, \bar{x}^{\prime}}$ is flat over $\mathcal{O}_{X, \bar{x}}$. Therefore $u$ is the identity in $\mathcal{O}_{X, \bar{x}}$.

Let $I$ be an ideal of $A$ with $I^{2}=\{0\}$, and $B=A / I$. Next we consider a case where $\rho$ is given by the natural homomorphism $A \rightarrow B$.

Claim 4.1.2. We assume that (i) $k=A / m$ is algebraically closed and (ii) there are a fine and sharp monoid $Q$ and a homomorphism $\pi_{Q}: Q \rightarrow M_{S, s}$ such that $Q \rightarrow M_{S, \bar{s}} \rightarrow \bar{M}_{S, \bar{s}}$ is bijective. If $h_{S^{\prime}, \bar{x}^{\prime}}=h_{S^{\prime}, \bar{x}^{\prime}}^{\prime}$ for all closed points $x^{\prime}$ lying over $s^{\prime}$, then $h_{\bar{x}}=h_{\bar{x}}^{\prime}$ for all closed points $x$ lying over $s$. 
Let $x$ be a closed point of $X$ lying over $s$, and $y=\phi(x)$. First of all, by [6] or Ogus' paper [8], there are fine and sharp monoids $P$ and $P^{\prime}$ and homomorphisms $P \rightarrow M_{X, \bar{x}}, Q \rightarrow P, P^{\prime} \rightarrow M_{Y, \bar{y}}, Q \rightarrow P^{\prime}$ with the following properties:

(1) The induced homomorphisms $P \rightarrow M_{X, \bar{x}} \rightarrow \bar{M}_{X, \bar{x}}$ and $P^{\prime} \rightarrow M_{Y, \bar{y}} \rightarrow$ $\bar{M}_{Y, \bar{y}}$ are bijective.

(2) The following diagrams are commutative:

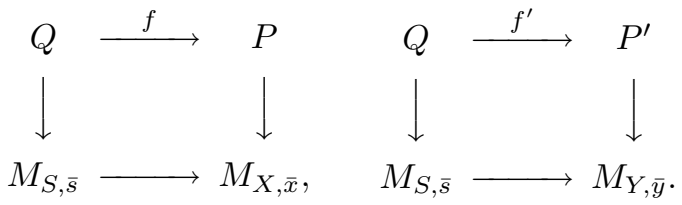

(3) There are étale neighborhoods $\left(U, x^{\prime}\right)$ and $\left(V, y^{\prime}\right)$ of $x$ and $y$ such that $P \rightarrow M_{X, \bar{x}}$ and $P^{\prime} \rightarrow M_{Y, \bar{y}}$ are defined over $U$ and $V$ respectively, and that the natural morphisms

$$
U \rightarrow \operatorname{Spec}\left(A \otimes_{A[Q]} A[P]\right) \quad \text { and } \quad V \rightarrow \operatorname{Spec}\left(A \otimes_{A[Q]} A\left[P^{\prime}\right]\right)
$$

are smooth at $x^{\prime}$ and $y^{\prime}$ respectively.

Clearly we may assume that $P, P^{\prime}$ and $Q$ are submonoids of $M_{X, \bar{x}}, M_{Y, \bar{y}}$ and $M_{S, \bar{s}}$ respectively. We set $U_{s}=U \times_{S} \operatorname{Spec}(\kappa(s)), V_{s}=V \times_{S} \operatorname{Spec}(\kappa(s))$, $\phi_{s}=\phi \times_{S} \operatorname{id}_{\operatorname{Spec}(\kappa(s))}, M_{U_{s}}=\left.M_{X}\right|_{U_{s}}, M_{V_{s}}=\left.M_{Y}\right|_{V_{s}}$ and $M_{k}=\left.M_{S}\right|_{\operatorname{Spec}(\kappa(s))}$. By Lemma 4.3 below, the admissibility of $\phi_{s}$ guarantees that for any irreducible components $T$ of $U_{s}, \phi_{s}(T) \nsubseteq \operatorname{Supp}\left(M_{V_{s}} / M_{k}\right)$.

Let $\sigma$ (resp. $\sigma^{\prime}$ ) be the set of all irreducible elements of $P$ not lying in $f(Q)$ (resp. the set of all irreducible elements of $P^{\prime}$ not lying in $f^{\prime}(Q)$ ). For $j \in \sigma$ and $i \in \sigma^{\prime}$, we denote $\alpha(j)$ by $x_{j}$ and $\alpha^{\prime}(i)$ by $y_{i}$, where $\alpha: M_{X, \bar{x}} \rightarrow \mathcal{O}_{X, \bar{x}}$ and $\alpha^{\prime}: M_{Y, \bar{y}} \rightarrow \mathcal{O}_{Y, \bar{y}}$ are the canonical homomorphisms. Moreover $\left.x_{j}\right|_{U_{s}}$ and $\left.y_{i}\right|_{V_{s}}$ are denoted by $x_{j s}$ and $y_{i s}$ respectively. Let us consider $h$ and $h^{\prime}$ on the fibers $X_{s}=X \times{ }_{S} \operatorname{Spec}(\kappa(s))$ and $Y_{s}=Y \times{ }_{S} \operatorname{Spec}(\kappa(s))$ over $s$. Using [6, Theorem 4.1], $\bar{h}_{\bar{x}}=\bar{h}_{\bar{x}}^{\prime}$ as homomorphisms $P^{\prime} \rightarrow P$. Thus we can set as follows:

$$
h_{\bar{x}}(i)=u_{i} \cdot\left(I_{i} \cdot \sigma+f\left(q_{i}\right)\right) \text { and } h_{\bar{x}}^{\prime}(i)=u_{i}^{\prime} \cdot\left(I_{i} \cdot \sigma+f\left(q_{i}\right)\right),
$$

where $q_{i} \in Q, I_{i} \in \mathbb{N}^{\sigma}$ and $u_{i}, u_{i}^{\prime} \in \mathcal{O}_{X, \bar{x}}^{\times}$. Then we have

$$
\phi^{*}\left(y_{i}\right)=\beta\left(q_{i}\right) \cdot x^{I_{i}} \cdot u_{i}=\beta\left(q_{i}\right) \cdot x^{I_{i}} \cdot u_{i}^{\prime},
$$


where $\beta: M_{S, \bar{s}} \rightarrow \mathcal{O}_{S, \bar{s}}$ is the canonical homomorphism. We claim the following:

$$
\text { If } \phi_{s}^{*}\left(y_{i s}\right) \neq 0 \text { for some } i \in \sigma^{\prime} \text {, then } q_{i}=0 \text { and } \phi^{*}\left(y_{i}\right)=x^{I_{i}} \cdot u_{i}=x^{I_{i}} \cdot u_{i}^{\prime} \text {. }
$$

Indeed, by (4.1.4), $\phi_{s}^{*}\left(y_{i s}\right)=\beta_{s}\left(q_{i}\right) \cdot x_{s}^{I_{i}} \cdot u_{i s}$ on $U_{s}$, where $\beta_{s}: Q \rightarrow k$ is a homomorphism given by

$$
\beta_{s}(q)= \begin{cases}1 & \text { if } q=0 \\ 0 & \text { otherwise }\end{cases}
$$

and $u_{i s}=\left.u_{i}\right|_{U_{s}}$. Thus $q_{i}=0$, which yields $\phi^{*}\left(y_{i}\right)=x^{I_{i}} \cdot u_{i}=x^{I_{i}} \cdot u_{i}^{\prime}$.

Here we consider the following four cases:

(A) $f: Q \rightarrow P$ splits and $f^{\prime}: Q \rightarrow P^{\prime}$ splits.

(B) $f: Q \rightarrow P$ does not split and $f^{\prime}: Q \rightarrow P^{\prime}$ splits.

(C) $f: Q \rightarrow P$ splits and $f^{\prime}: Q \rightarrow P^{\prime}$ does not split.

(D) $f: Q \rightarrow P$ does not split and $f^{\prime}: Q \rightarrow P^{\prime}$ does not split.

(Case A): In this case, there are submonoids $N$ and $N^{\prime}$ of $P$ and $P^{\prime}$ respectively such that $P=f(Q) \times N$ and $P^{\prime}=f^{\prime}(Q) \times N^{\prime}$. Note that $\sigma$ and $\sigma^{\prime}$ are nothing more than the set of all irreducible elements of $N$ and $N^{\prime}$ respectively. Then, by the local structure theorem (cf. Theorem 3.1),

$$
\operatorname{Supp}\left(M_{V_{s}} / M_{k}\right)=\bigcup_{i \in \sigma^{\prime}}\left\{y_{i s}=0\right\} .
$$

around $y^{\prime}$ on $V_{s}$. Thus, using the admissibility of $\phi_{s}, \phi_{s}^{*}\left(y_{i s}\right) \neq 0$. Hence, by (4.1.5), $q_{i}=0$ and $x^{I_{i}} \cdot u_{i}=x^{I_{i}} \cdot u_{i}^{\prime}$ for all $i \in \sigma^{\prime}$. Therefore $u_{i}=u_{i}^{\prime}$ for all $i \in \sigma^{\prime}$ because $x_{j}$ 's are regular elements (cf. Theorem 3.1).

(Case B): In this case, there is a submonoid $N^{\prime}$ of $P^{\prime}$ such that $P^{\prime}=$ $f^{\prime}(Q) \times N^{\prime}$. Moreover $P$ is of semistable type

$$
\left(\sigma, q_{0}, \Delta, B\right)
$$

over $Q$ for some $q_{0} \in Q$ and $\Delta, B \in \mathbb{N}^{\sigma}$. By the local structure theorem (cf. Theorem 3.1),

$$
\operatorname{Supp}\left(M_{V_{s}} / M_{k}\right)=\bigcup_{i \in \sigma^{\prime}}\left\{y_{i s}=0\right\}
$$


around $y^{\prime}$ on $V_{s}$. Thus, by the admissibility of $\phi_{s}, \phi_{s}^{*}\left(y_{i s}\right) \neq 0$. Therefore, by (4.1.5), $q_{i}=0$ and $\phi^{*}\left(y_{i}\right)=x^{I_{i}} \cdot u_{i}=x^{I_{i}} \cdot u_{i}^{\prime}$ for all $i \in \sigma^{\prime}$. Since $U_{s}$ is given by $\prod_{j \in \operatorname{Supp}(\Delta)} x_{j s}=0$, if $j \in \operatorname{Supp}\left(I_{i}\right) \cap \operatorname{Supp}(\Delta)$, then $\phi_{s}^{*}\left(y_{i s}\right)=0$ on the irreducible component $\left\{x_{j s}=0\right\}$ of $U_{s}$. This contradicts to the admissibility of $\phi_{s}$. Hence $\operatorname{Supp}\left(I_{i}\right) \cap \operatorname{Supp}(\Delta)=\emptyset$ for all $i \in \sigma^{\prime}$. Thus $x^{I_{i}}$ 's are regular elements (cf. Theorem 3.1). Therefore $u_{i}=u_{i}^{\prime}$ for all $i \in \sigma^{\prime}$.

(Case C): In this case, there is a submonoid $N$ of $P$ with $P=f(Q) \times N$. $P^{\prime}$ is of semistable type

$$
\left(\sigma^{\prime}, q_{0}^{\prime}, \Delta^{\prime}, B^{\prime}\right)
$$

over $Q$ for some $q_{0}^{\prime} \in Q$ and $\Delta^{\prime}, B^{\prime} \in \mathbb{N}^{\sigma^{\prime}}$. Note that

$$
\operatorname{Supp}\left(M_{V_{s}} / M_{k}\right)=\operatorname{Sing}\left(V_{s}\right) \cup \bigcup_{i \in \sigma^{\prime} \backslash \operatorname{Supp}\left(\Delta^{\prime}\right)}\left\{y_{i s}=0\right\} .
$$

around $y^{\prime}$ on $V_{s}$ (cf. Theorem 3.1).

Let us see that if $\phi_{s}^{*}\left(y_{i s}\right) \neq 0$ for some $i \in \sigma^{\prime}$, then $q_{i}=0$ and $u_{i}=u_{i}^{\prime}$. Indeed, by (4.1.5), we have $q_{i}=0$ and $x^{I_{i}} \cdot u_{i}=x^{I_{i}} \cdot u_{i}^{\prime}$. Thus $u_{i}=u_{i}^{\prime}$ because $x^{I_{i}}$ 's are regular elements (cf. Theorem 3.1).

Therefore we may assume that there is $i_{0} \in \sigma^{\prime}$ with $\phi_{s}^{*}\left(y_{i_{0} s}\right)=0$. By using the admissibility of $\phi_{s}, \phi_{s}^{*}\left(y_{i s}\right) \neq 0$ for $i \in \sigma^{\prime} \backslash \operatorname{Supp}\left(\Delta^{\prime}\right)$. Thus $i_{0} \in \operatorname{Supp}\left(\Delta^{\prime}\right)$. Moreover, if $\phi_{s}^{*}\left(y_{i_{1} s}\right)=0$ for $i_{1} \in \operatorname{Supp}\left(\Delta^{\prime}\right) \backslash\left\{i_{0}\right\}$, then

$$
\phi_{s}\left(U_{s}\right) \subseteq\left\{y_{i_{0} s}=y_{i_{1} s}=0\right\} \subseteq \operatorname{Sing}\left(V_{s}\right),
$$

which contradicts to the admissibility of $\phi_{s}$. Thus $\phi_{s}^{*}\left(y_{i s}\right) \neq 0$ for all $i \in$ $\sigma^{\prime} \backslash\left\{i_{0}\right\}$. Hence $u_{i}=u_{i}^{\prime}$ for all $i \in \sigma^{\prime} \backslash\left\{i_{0}\right\}$. Let us consider the relation

$$
\Delta^{\prime} \cdot \sigma^{\prime}=f^{\prime}\left(q_{0}^{\prime}\right)+B^{\prime} \cdot \sigma^{\prime} .
$$

Then we have

$$
\left\{\begin{array}{l}
\sum_{i \in \operatorname{Supp}\left(\Delta^{\prime}\right)} h_{\bar{x}}(i)=f^{\prime}\left(q_{0}^{\prime}\right)+\sum_{i \in \operatorname{Supp}\left(B^{\prime}\right)} B^{\prime}(i) h_{\bar{x}}(i), \\
\sum_{i \in \operatorname{Supp}\left(\Delta^{\prime}\right)} h_{\bar{x}}^{\prime}(i)=f^{\prime}\left(q_{0}^{\prime}\right)+\sum_{i \in \operatorname{Supp}\left(B^{\prime}\right)} B^{\prime}(i) h_{\bar{x}}^{\prime}(i) .
\end{array}\right.
$$

Here $h_{\bar{x}}(i)=h_{\bar{x}}^{\prime}(i)$ for all $i \neq i_{0}$. Thus we can see that $h_{\bar{x}}\left(i_{0}\right)=h_{\bar{x}}^{\prime}\left(i_{0}\right)$.

(Case D): In the final case, $P$ and $P^{\prime}$ are of semistable type

$$
\left(\sigma, q_{0}, \Delta, B\right) \quad \text { and } \quad\left(\sigma^{\prime}, q_{0}^{\prime}, \Delta^{\prime}, B^{\prime}\right)
$$

over $Q$ for some $q_{0}, q_{0}^{\prime} \in Q, \Delta, B \in \mathbb{N}^{\sigma}$ and $\Delta^{\prime}, B^{\prime} \in \mathbb{N}^{\sigma^{\prime}}$. For $j \in \operatorname{Supp}(\Delta)$ and $i \in \operatorname{Supp}\left(\Delta^{\prime}\right)$, let $U_{j s}$ and $V_{i s}$ be the irreducible components of $U_{s}$ and $V_{s}$ 
given by $x_{j s}=0$ and $y_{i s}=0$ respectively. By the admissibility of $\phi_{s}$, for each $j \in \operatorname{Supp}(\Delta)$, there is a unique $i \in \operatorname{Supp}\left(\Delta^{\prime}\right)$ with $\phi_{s}\left(U_{j s}\right) \subseteq V_{i s}$. This $i$ is denoted by $\mu(j)$. Note that

$$
\operatorname{Supp}\left(M_{V_{s}} / M_{k}\right)=\operatorname{Sing}\left(V_{s}\right) \cup \bigcup_{i \in \sigma^{\prime} \backslash \operatorname{Supp}\left(\Delta^{\prime}\right)}\left\{y_{i s}=0\right\}
$$

around $y^{\prime}$ on $V_{s}$. Here we claim the following:

(i) If $i \neq \mu(j)$ for $i \in \sigma^{\prime}$ and $j \in \operatorname{Supp}(\Delta)$, then $\left.\phi^{*}\left(y_{i}\right)\right|_{U_{j s}} \neq 0$.

(ii) If there is $j \in \operatorname{Supp}(\Delta)$ with $i \neq \mu(j)$, then $q_{i}=0$ and $\phi^{*}\left(y_{i}\right)=x^{I_{i}} \cdot u_{i}=$ $x^{I_{i}} \cdot u_{i}^{\prime}$.

(iii) If $i \notin \mu(\operatorname{Supp}(\Delta))$, then $q_{i}=0$ and $u_{i}=u_{i}^{\prime}$.

(iv) If $i, i^{\prime} \in \operatorname{Supp}\left(\Delta^{\prime}\right)$ and $i \neq i^{\prime}$, then $\operatorname{Supp}\left(I_{i}\right) \cap \operatorname{Supp}\left(I_{i^{\prime}}\right)=\emptyset$.

(i) is obvious by the admissibility of $\phi_{s}$. (ii) is a consequence of (i) and (4.1.5). Let us see (iii). By (ii), $q_{i}=0$ and $\phi^{*}\left(y_{i}\right)=x^{I_{i}} \cdot u_{i}=x^{I_{i}} \cdot u_{i}^{\prime}$. Using (i), $\left.\phi^{*}\left(y_{i}\right)\right|_{U_{j s}} \neq 0$ for all $j \in \operatorname{Supp}(\Delta)$. Thus $\operatorname{Supp}\left(I_{i}\right) \cap \operatorname{Supp}(\Delta)=\emptyset$. Hence $x^{I_{i}}$ is a regular element (cf. Theorem 3.1). Therefore $u_{i}=u_{i}^{\prime}$. Finally we consider (iv). We assume that there is a $j \in \operatorname{Supp}\left(I_{i}\right) \cap \operatorname{Supp}\left(I_{i^{\prime}}\right)$. Then, since $\phi^{*}\left(y_{l}\right)=\beta\left(q_{l}\right) \cdot x^{I_{l}} \cdot u_{l}$ for all $l \in \sigma^{\prime}$,

$$
\phi\left(U_{j s}\right) \subseteq\left\{y_{i s}=y_{i^{\prime} s}=0\right\} \subseteq \operatorname{Sing}\left(V_{s}\right),
$$

which contradicts to the admissibility of $\phi_{s}$.

Let us start the proof of the case (D). First we consider the case where $\# \mu(\operatorname{Supp}(\Delta))=1$, i.e., $\mu(\operatorname{Supp}(\Delta))=\left\{i_{0}\right\}$ for some $i_{0} \in \operatorname{Supp}\left(\Delta^{\prime}\right)$. Then, by (iii), for $i \neq i_{0}, q_{i}=0$ and $u_{i}=u_{i}^{\prime}$. Considering the relation:

$$
\Delta^{\prime} \cdot \sigma^{\prime}=f^{\prime}\left(q_{0}^{\prime}\right)+B^{\prime} \cdot \sigma^{\prime},
$$

we have

$$
\left\{\begin{array}{l}
\sum_{i \in \operatorname{Supp}\left(\Delta^{\prime}\right)} h_{\bar{x}}(i)=f^{\prime}\left(q_{0}^{\prime}\right)+\sum_{i \in \operatorname{Supp}\left(B^{\prime}\right)} B^{\prime}(i) h_{\bar{x}}(i), \\
\sum_{i \in \operatorname{Supp}\left(\Delta^{\prime}\right)} h_{\bar{x}}^{\prime}(i)=f^{\prime}\left(q_{0}^{\prime}\right)+\sum_{i \in \operatorname{Supp}\left(B^{\prime}\right)} B^{\prime}(i) h_{\bar{x}}^{\prime}(i) .
\end{array}\right.
$$

Since $h_{\bar{x}}(i)=h_{\bar{x}}^{\prime}(i)$ for all $i \neq i_{0}$, we can see that $h_{\bar{x}}\left(i_{0}\right)=h_{\bar{x}}^{\prime}\left(i_{0}\right)$.

Next let us consider the case where $\# \mu(\operatorname{Supp}(\Delta)) \geq 2$. In this case, by (ii), $q_{i}=0$ and $\phi^{*}\left(y_{i}\right)=x^{I_{i}} \cdot u_{i}=x^{I_{i}} \cdot u_{i}^{\prime}$ for all $i \in \sigma^{\prime}$. Moreover, by (iii), $u_{i}=u_{i}^{\prime}$ 
for all $i \in \sigma^{\prime} \backslash \operatorname{Supp}\left(\Delta^{\prime}\right)$. By our assumption, $u_{i} \equiv u_{i}^{\prime} \bmod I \mathcal{O}_{X, \bar{x}}$. Note that $x_{j}(j \notin \operatorname{Supp}(\Delta))$ is regular. Thus, if we set $I_{i}^{\prime}=\left.I_{i}\right|_{\operatorname{Supp}(\Delta)} \in \mathbb{N}^{\operatorname{Supp}(\Delta)}$, then

$$
x^{I_{i}^{\prime}} \cdot u_{i}=x^{I_{i}^{\prime}} \cdot u_{i}^{\prime}
$$

for all $i \in \operatorname{Supp}\left(\Delta^{\prime}\right)$. By (iv), $\operatorname{Supp}\left(I_{i}^{\prime}\right) \cap \operatorname{Supp}\left(I_{i^{\prime}}^{\prime}\right)=\emptyset$ for all $i \neq i^{\prime} \in \operatorname{Supp}\left(\Delta^{\prime}\right)$. Further let us consider the relation

$$
\Delta^{\prime} \cdot \sigma^{\prime}=f^{\prime}\left(q_{0}^{\prime}\right)+B^{\prime} \cdot \sigma^{\prime} .
$$

Since $h_{\bar{x}}(i)=h_{\bar{x}}^{\prime}(i)$ for all $i \in \sigma^{\prime} \backslash \operatorname{Supp}\left(\Delta^{\prime}\right)$, we have

$$
\sum_{i \in \operatorname{Supp}\left(\Delta^{\prime}\right)} h_{\bar{x}}(i)=\sum_{i \in \operatorname{Supp}\left(\Delta^{\prime}\right)} h_{\bar{x}}^{\prime}(i),
$$

which implies $\prod_{i \in \operatorname{Supp}\left(\Delta^{\prime}\right)} u_{i}=\prod_{i \in \operatorname{Supp}\left(\Delta^{\prime}\right)} u_{i}^{\prime}$. Here we set $v_{i}=u_{i} / u_{i}^{\prime}$ for $i \in \operatorname{Supp}\left(\Delta^{\prime}\right)$. Then, gathering the above observations, we have seen that

$$
\left\{\begin{array}{l}
x^{I_{i}^{\prime}}=x^{I_{i}^{\prime}} \cdot v_{i} \text { for all } i \in \operatorname{Supp}\left(\Delta^{\prime}\right), \\
v_{i} \equiv 1 \bmod I \mathcal{O}_{X, \bar{x}} \text { for all } i \in \operatorname{Supp}\left(\Delta^{\prime}\right), \\
\prod_{i \in \operatorname{Supp}\left(\Delta^{\prime}\right)} v_{i}=1, \\
\operatorname{Supp}\left(I_{i}^{\prime}\right) \cap \operatorname{Supp}\left(I_{i^{\prime}}^{\prime}\right)=\emptyset \text { for all } i \neq i^{\prime} \in \operatorname{Supp}\left(\Delta^{\prime}\right) .
\end{array}\right.
$$

Since $U \rightarrow \operatorname{Spec}\left(A \otimes_{A[Q]} A[P]\right)$ is smooth at $x^{\prime}, U \rightarrow \operatorname{Spec}\left(A \otimes_{A[Q]} A\left[P \times \mathbb{N}^{e}\right]\right)$ is étale at $x^{\prime}$ for some $e \geq 0$. Let $o$ be the origin of $\operatorname{Spec}\left(A \otimes_{A[Q]} A\left[P \times \mathbb{N}^{e}\right]\right)$. Then the residue field of $A \otimes_{A[Q]} A\left[P \times \mathbb{N}^{e}\right]$ at $o$ is $k$. Moreover the residue fields of $\mathcal{O}_{U, x^{\prime}}$ and $\mathcal{O}_{X, \bar{x}}$ are $k$ because $k$ is algebraically closed. Therefore the completion of $A \otimes_{A[Q]} A\left[P \times \mathbb{N}^{e}\right]$ at $o$ is isomorphic to the completion of $\mathcal{O}_{X, \bar{x}}$. Thus, by Lemma 4.4 below, $v_{i}=1$, that is, $u_{i}=u_{i}^{\prime}$ for all $i \in \operatorname{Supp}\left(\Delta^{\prime}\right)$. Hence we complete the proof of 4.1.2.

Let us start the proof of 4.1. Let $k=A / m$ and $\bar{k}$ the algebraic closure of $k$. By virtue of [3, EGA III, Chapter 0, 10.3.1], there are a noetherian local ring $(B, n)$ and a local homomorphism $A \rightarrow B$ such that $m B=n, B / n$ is isomorphic to $\bar{k}$ over $k=A / m$ and that $B$ is flat over $A$. Thus, by Claim 4.1.1, we may assume that the residue field $k=A / m$ is algebraically closed. Moreover, by Proposition 2.1.1, we may further assume that there are a fine and sharp monoid $Q$ and a homomorphism $\pi_{Q}: Q \rightarrow M_{S, s}$ such that $Q \rightarrow M_{S, \bar{s}} \rightarrow \bar{M}_{S, \bar{s}}$ is bijective.

Let $A_{i}=A / m^{i+1}, \rho_{i}: A_{i} \rightarrow A_{i-1}$ the canonical homomorphism and $I_{i}=\operatorname{Ker}\left(\rho_{i}\right)$. Then $A_{0}=k$ and $I_{i}^{2}=\{0\}$ for $i \geq 1$. We set $X_{i}=X \times{ }_{S} \operatorname{Spec}\left(A_{i}\right)$, 
$M_{X_{i}}=\left.M_{X}\right|_{X_{i}}, Y_{i}=Y \times_{S} \operatorname{Spec}\left(A_{i}\right), M_{Y_{i}}=\left.M_{Y}\right|_{Y_{i}}$. Moreover the induced two morphisms $M_{Y_{i}} \rightarrow M_{X_{i}}$ via $h$ and $h^{\prime}$ are denoted by $h_{i}$ and $h_{i}^{\prime}$ respectively. Note that $h_{0}=h_{0}^{\prime}$ at any closed points of $X_{s}$ by [6]. By Claim 4.1.2, $h_{n}=h_{n}^{\prime}$ at any closed points lying over $s$ implies that $h_{n+1}=h_{n+1}^{\prime}$ at any closed points lying over $s$. Therefore we have $h_{n}=h_{n}^{\prime}$ at any closed points of $X_{s}$ for all $n \geq 0$. Let $x$ be a closed point of $X$ over $s$ and $y=\phi(x)$. Since $\bar{h}_{\bar{x}}=\bar{h}_{\bar{x}}^{\prime}$ as a homomorphism $\bar{M}_{Y, \bar{y}} \rightarrow \bar{M}_{X, \bar{x}}$, for $w \in M_{Y, \bar{y}}$, there is $u \in \mathcal{O}_{X, \bar{x}}^{\times}$with $h_{\bar{x}}(w)=h_{\bar{x}}^{\prime}(w) \cdot u$. Since $h_{n}=h_{n}^{\prime}$, we can see that $u-1 \in m^{n+1} \mathcal{O}_{X, \bar{x}}$. Note that $\mathcal{O}_{X, \bar{x}}$ is noetherian, which implies that $\bigcap_{n=0} m^{n+1} \mathcal{O}_{X, \bar{x}}=\{0\}$. Therefore $u=1$.

As corollary of Theorem 4.1, we have the following:

Corollary 4.2 (Rigidity theorem). Let $f: X \rightarrow S$ and $g: Y \rightarrow S$ be semistable schemes over a locally noetherian scheme $S$, and let $\phi: X \rightarrow Y$ be a morphism over $S$. Let $M_{X}, M_{Y}$ and $M_{S}$ be fine log structures on $X, Y$ and $S$ respectively. We assume that $\left(X, M_{X}\right)$ and $\left(Y, M_{Y}\right)$ are log smooth and integral over $\left(S, M_{S}\right)$ and $\phi$ is admissible with respect to $M_{Y} / M_{S}$. If we have log morphisms

$$
(\phi, h):\left(X, M_{X}\right) \rightarrow\left(Y, M_{Y}\right) \quad \text { and } \quad\left(\phi, h^{\prime}\right):\left(X, M_{X}\right) \rightarrow\left(Y, M_{Y}\right)
$$

over $\left(S, M_{S}\right)$ as extensions of $\phi: X \rightarrow Y$, then $h=h^{\prime}$.

The following two lemmas were needed for the proof of Theorem 4.1.

Lemma 4.3. Let

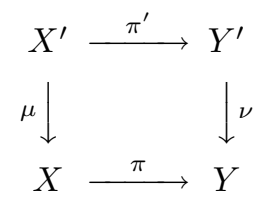

be a commutative diagram of reduced algebraic schemes over an algebraically closed field such that $X$ and $X^{\prime}$ are equi-dimensional and $\mu$ is flat. Let $Z$ be a closed subset of $Y$. If $\pi(T) \nsubseteq Z$ for any irreducible components $T$ of $X$, then $\pi^{\prime}\left(T^{\prime}\right) \nsubseteq \nu^{-1}(Z)$ for any irreducible components $T^{\prime}$ of $X^{\prime}$.

Proof. We assume that $\pi^{\prime}\left(T^{\prime}\right) \subseteq \nu^{-1}(Z)$ for an irreducible component $T^{\prime}$ of $X^{\prime}$. Then

$$
\pi\left(\mu\left(T^{\prime}\right)\right)=\nu\left(\pi^{\prime}\left(T^{\prime}\right)\right) \subseteq \nu\left(\nu^{-1}(Z)\right) \subseteq Z .
$$

Let $T$ be the Zariski closure of $\mu\left(T^{\prime}\right)$. If $\operatorname{dim} T<\operatorname{dim} X$, then

$$
\operatorname{dim} \mu^{-1}(x) \geq \operatorname{dim} T^{\prime}-\operatorname{dim} T>\operatorname{dim} X^{\prime}-\operatorname{dim} X
$$


for $x \in \mu\left(T^{\prime}\right)$, which is a contradiction because $\mu$ is flat. Thus we have $\operatorname{dim} T=$ $\operatorname{dim} X$, which means that $T$ is an irreducible component of $X$. On the other hand, we know $\pi(T) \subseteq Z$. This is a contradiction to our assumption. Therefore we get our lemma.

Lemma 4.4. Let $(A, m)$ be a noetherian complete local ring and $A \llbracket X_{1}$, $\ldots, X_{n} \rrbracket$ the ring of formal power series of n-variables over $A$. For a fixed $a \in m$, let

$$
R=A \llbracket X_{1}, \ldots, X_{n} \rrbracket /\left(X_{1} \cdots X_{n}-a\right)
$$

and $J$ an ideal of $R$ with $J^{2}=0$. Let $u_{1}, \ldots, u_{l}$ be elements of $R$ and $I_{1}, \ldots, I_{l}$ elements of $\mathbb{N}^{n}$ with $\operatorname{Supp}\left(I_{i}\right) \cap \operatorname{Supp}\left(I_{j}\right)=\emptyset$ for $i \neq j$. We assume that (1) $u_{1} \cdots u_{l}=1$, (2) $X^{I_{i}} u_{i}=X^{I_{i}}$ in $R$ for all $i$, and that $(3) u_{i} \equiv 1 \bmod J$. Then we have $u_{1}=\cdots=u_{l}=1$.

Proof. We set $\Sigma=\left\{I \in \mathbb{N}^{n} \mid \Delta \not \leq I\right\}$ and

$$
A \llbracket X_{1}, \ldots, X_{n} \rrbracket_{\Sigma}=\left\{\sum_{I \in \Sigma} a_{I} X^{I} \mid a_{I} \in A\right\},
$$

where $\Delta=(1, \ldots, 1)$. Then, by Lemma 1.1 .2 , the natural map $A \llbracket X_{1}, \ldots, X_{n} \rrbracket_{\Sigma}$ $\rightarrow R$ is bijective. Here we claim the following:

Claim 4.4.1. Let $T$ be an element of $\mathbb{N}^{n}$. We set $\Sigma_{T}=\{I \in \Sigma \mid$ $I+T \geq \Delta\}$. Then, for $f \in A \llbracket X_{1}, \ldots, X_{n} \rrbracket_{\Sigma}$, if $X^{T} f=0$ in $R$, then $f$ can be written by a form

$$
f=\sum_{I \in \Sigma_{T}} b_{I} X^{I}
$$

If either $T=(0, \ldots, 0)$ or $T \geq \Delta$, then our assertion is trivial. Thus we may assume that $T \neq(0, \ldots, 0)$ and $T \nsupseteq \Delta$. For $I \in \mathbb{N}^{n}$, we can find a nonnegative integer $\alpha$ and $J \in \Sigma$ with $I=\alpha \Delta+J$. We denote $\alpha$ and $J$ by $\alpha(I)$ and $J(I)$ respectively. Here let us see that $J(I+T) \notin\left\{S+T \mid S \in \Sigma \backslash \Sigma_{T}\right\}$ for $I \in \Sigma_{T}$. Indeed, since $I \in \Sigma_{T}$, we can find $i$ with $I(i)=0$ and $T(i)>0$. Thus

$$
J(I+T)(i)=T(i)-\alpha(I+T)<T(i) .
$$

Hence $J(I+T) \notin\left\{S+T \mid S \in \Sigma \backslash \Sigma_{T}\right\}$.

We set $f=\sum_{I \in \Sigma} a_{I} X^{I}$. Then

$$
\begin{aligned}
X^{T} f & =\sum_{I \in \Sigma_{T}} a_{I} X^{I+T}+\sum_{I \in \Sigma \backslash \Sigma_{T}} a_{I} X^{I+T} \\
& =\sum_{I \in \Sigma_{T}} a_{I} a^{\alpha(I+T)} X^{J(I+T)}+\sum_{I \in \Sigma \backslash \Sigma_{T}} a_{I} X^{I+T} .
\end{aligned}
$$


Thus $a_{I}=0$ for $I \in \Sigma \backslash \Sigma_{T}$, which complete the proof of 4.4.1.

Since $u_{i} \equiv 1 \bmod J$, there is $a_{i} \in J$ with $u_{i}=1+a_{i}$. Then $X^{I_{i}} a_{i}=0$. Moreover, since $J^{2}=0$,

$$
u_{1} \cdots u_{l}=1+a_{1}+\cdots+a_{l}=1
$$

Hence $a_{1}+\cdots+a_{l}=0$. Since $X^{I_{i}} a_{i}=0$, by the above claim, $a_{i}=\sum_{I \in \Sigma_{I_{i}}} c_{i, I} X^{I}$, where $\Sigma_{I_{i}}=\left\{I \in \Sigma \mid I+I_{i} \geq \Delta\right\}$. Therefore

$$
\sum_{i=1}^{l} \sum_{I \in \Sigma_{I_{i}}} c_{i, I} X^{I}=0 .
$$

Note that if $I \in \Sigma_{I_{i}}$ and $I^{\prime} \in \Sigma_{I_{j}}$ for $i \neq j$, then $I \neq I^{\prime}$ because $\operatorname{Supp}\left(I_{i}\right) \cap$ $\operatorname{Supp}\left(I_{j}\right)=\emptyset$. Thus we can see that $c_{i, I}=0$, which shows us $a_{i}=0$ for all $i$.

\section{References}

[1] M. Artin, Algebraic approximation of structures over complete local rings, Inst. Hautes Études Sci. Publ. Math. No. 36 (1969), 23-58.

[2] S. U. Chase, Direct products of modules, Trans. Amer. Math. Soc. 97 (1960), 457-473.

[3] A. Grothendieck, Éléments de géométrie algébrique, III. Étude cohomologique des faisceaux cohérents. I, Inst. Hautes Études Sci. Publ. Math. No. 11 (1961), 167 pp.

[4] K. Kato, Logarithmic structures of Fontaine-Illusie, in Algebraic analysis, geometry, and number theory (Baltimore, MD, 1988), 191-224, Johns Hopkins Univ. Press, Baltimore, MD.

[5] S. Kobayashi and T. Ochiai, Meromorphic mappings onto compact complex spaces of general type, Invent. Math. 31 (1975), no. 1, 7-16.

[6] I. Iwanari and A. Moriwaki, Dominant rational maps in the category of log schemes, to appear in Tohoku Math. J. 59 (2007).

7] A. Moriwaki, Descent of log morphisms, in preparation.

[8] A. Ogus, Logarithmic De Rham cohomology, preprint (1997).

[9] M. C. Olsson, Universal log structures on semi-stable varieties, Tohoku Math. J. (2) 55 (2003), no. 3, 397-438. 